\title{
On Rosenau-Type Approximations to Fractional Diffusion Equations
}

\author{
G.Furioli * \\ A. Pulvirenti ${ }^{\dagger}$ \\ E. Terraneo $\ddagger$ \\ G. Toscani ${ }^{\S}$
}

July 9, 2018

\begin{abstract}
Owing to the Rosenau argument Ros92, originally proposed to obtain a regularized version of the Chapman-Enskog expansion of hydrodynamics, we introduce a non-local linear kinetic equation which approximates a fractional diffusion equation. We then show that the solution to this approximation, apart of a rapidly vanishing in time perturbation, approaches the fundamental solution of the fractional diffusion (a Lévy stable law) at large times.
\end{abstract}

2000 AMS subject classification. 35K55, 35K60, 35K65, 35B40.

Key words. Fractional diffusion equations, non-local models, Fourier metrics, Rosenau approximation, Lévy-type distributions.

\section{Introduction}

In Ros92, Rosenau proposed a regularized version of the Chapman-Enskog expansion of hydrodynamics, with a suitably modified viscosity term. This model is given by the scalar equation

$$
\partial_{t} f(v, t)+\partial_{v} \Psi(f)(v, t)=\mathcal{D}_{\varepsilon} f(v, t), \quad v \in \mathbb{R}, t \geq 0
$$

where $\varepsilon \ll 1$ is a small positive parameter,

$$
\widehat{\mathcal{D}_{\varepsilon} f}(\xi, t)=\frac{-\varepsilon \xi^{2}}{1+\varepsilon^{2} m^{2} \xi^{2}} \widehat{f}(\xi, t),
$$

and $\widehat{g}(\xi)$ denotes the Fourier transform of $g(v)$

$$
\mathcal{F} g(\xi)=\widehat{g}(\xi)=\int_{\mathbb{R}} g(v) \mathrm{e}^{-i v \xi} \mathrm{d} v, \quad \xi \in \mathbb{R} .
$$

The operator on the right hand side looks like the usual viscosity term $\varepsilon f_{v v}$ at low wave-numbers $\xi$, while for higher wave numbers it is intended to model a bounded approximation of a linearized collision operator, thereby avoiding the artificial instabilities that occur when the Chapman-Enskog expansion for such an operator is truncated after a finite number of terms.

\footnotetext{
${ }^{*}$ University of Bergamo, viale Marconi 5, 24044 Dalmine, ITALY.

${ }^{\dagger}$ Department of Mathematics, University of Pavia, via Ferrata 1, 27100 Pavia, ITALY.

${ }^{\ddagger}$ Department of Mathematics, University of Milan, via Saldini 50, 20133 Milano, ITALY.

${ }^{\S}$ Department of Mathematics, University of Pavia, via Ferrata 1, 27100 Pavia, ITALY.
} 
Note that the right hand side of (1) can be written in the Fourier variable as

$$
\frac{-\varepsilon \xi^{2}}{1+\varepsilon^{2} m^{2} \xi^{2}} \widehat{f}(\xi, t)=\frac{\varepsilon}{(\varepsilon m)^{2}}\left(\frac{1}{1+\varepsilon^{2} m^{2} \xi^{2}} \widehat{f}(\xi, t)-\widehat{f}(\xi, t)\right)=\mathcal{F}\left(\frac{1}{m \bar{\varepsilon}}\left(N_{\bar{\varepsilon}} * f-f\right)\right)(\xi, t)
$$

where $\bar{\varepsilon}=m \varepsilon, *$ denotes convolution and

$$
N_{\gamma}(v)=\frac{1}{2 \gamma} \mathrm{e}^{-|v| / \gamma}
$$

is a non-negative function satisfying $\left\|N_{\gamma}\right\|_{L^{1}}=1$. In other words, the approximation proposed by Rosenau consists in substituting the linear diffusion equation

$$
\partial_{t} g(v, t)=\partial_{v v}^{2} g(v, t)
$$

with the linear non-local kinetic equation

$$
\partial_{t} g(v, t)=\frac{1}{\varepsilon^{2}}\left[N_{\varepsilon} * g(v, t)-g(v, t)\right]
$$

in which the background "Maxwellian" $N_{\varepsilon}$ is given by (2) Cer88, PT13.

The study of the main properties of the Rosenau approximation (11) and of its relaxation part (3) has attracted a lot of interest [LT01, ST92, RT13. Indeed, while it is clear that at fixed time the solution to (3) represents, for sufficiently small values of the $\varepsilon$ parameter, a good approximation of the solution to the heat equation, the characteristics of its solution for large times, and its possible similarities with that of the heat equation are not evident. This last problem has been recently addressed and studied in details in RT13.

In view of the recent results in [RT13, the argument proposed by Rosenau for the linear heat equation appears to be of high interest for further applications. Maybe the most natural extension of his idea is to apply a similar modification to other types of linear diffusion equations, like the fractional diffusion equations. Fractional in space diffusion equations share in fact with the linear diffusion a simple representation in Fourier variables, which is at the basis of the introduction of a suitable Rosenau approximation. However, while interesting for its possible applications, this type of approximation has not been studied so far.

Fractional in space diffusion equations appear in many contexts. Among others, the review paper by Klafter et al. KZS97 provides numerous references to physical phenomena in which these anomalous diffusion occurs (cf. Cha98, BWM00, GM98, SBMW01, MFL02, Vaz11] and the reference therein for various details on both mathematical and physical aspects).

As it can be argued from the original application, the Rosenau approximation establishes a clear connection between diffusion equations and non-local kinetic equations. Possible connections between Boltzmann type equations and fractional in space diffusion equations have been studied only recently in [MMM11, FPTT12. While the analysis of Mellet, Mischler and Mouhot in MMM11 is devoted to the study of linear kinetic equations of Boltzmann type, and their connection with fractional diffusion equations, the results in [FPTT12 refer to the nonlinear one dimensional Kac model for dissipative collisions introduced in [PT04, and to its grazing collision limit.

The fractional diffusion equations read

$$
\partial_{t} g(v, t)=-(\sqrt{-\Delta})^{\lambda} g(v, t),
$$

where $0<\lambda<2$. The fractional derivative operator $(\sqrt{-\Delta})^{\lambda}$ is defined in the Fourier variable as

$$
\mathcal{F}\left((\sqrt{-\Delta})^{\lambda} h\right)(\xi)=|\xi|^{\lambda} \widehat{h}(\xi)
$$

Similarly to (1), the Rosenau-type correction consists in substituting the fractional diffusion equation (in Fourier variable)

$$
\partial_{t} \widehat{g}(\xi, t)=-|\xi|^{\lambda} \widehat{g}(\xi, t)
$$


with the equation

$$
\partial_{t} \widehat{g}(\xi, t)=\frac{-|\xi|^{\lambda}}{1+|\varepsilon \xi|^{\lambda}} \widehat{g}(\xi, t)=\frac{1}{\varepsilon^{\lambda}}\left[\widehat{M}_{\lambda}(\varepsilon \xi) \widehat{g}(\xi, t)-\widehat{g}(\xi, t)\right]
$$

where $\varepsilon \ll 1$. Note that in this case the Maxwellian function is expressed in Fourier variable by

$$
\widehat{M}_{\lambda}(\xi)=\frac{1}{1+|\xi|^{\lambda}}
$$

It is notable that, for all $0<\lambda \leq 2$, this Maxwellian function is the characteristic function of a symmetric probability distribution known in probability theory with the name of Linnik distribution Lin53, Lin62. In addition, when $\lambda>1, \widehat{M}_{\lambda} \in L^{1}(\mathbb{R})$, which allows us to apply the inversion formula to conclude that $M_{\lambda}$ is a probability density function. Consequently, in the case of a fractional diffusion equation with $\lambda>1$, Rosenau approximation consists in substituting the fractional diffusion (4) with the non-local linear Boltzmann equation

$$
\partial_{t} g(v, t)=\frac{1}{\varepsilon^{\lambda}}\left[M_{\lambda, \varepsilon} * g(v, t)-g(v, t)\right]
$$

where the Maxwellian $M_{\lambda, \varepsilon}$ is defined through its Fourier transform by the formula $\widehat{M}_{\lambda, \varepsilon}(\xi)=\widehat{M}_{\lambda}(\varepsilon \xi)$. Unlikely we note that, on the contrary to what happens in the case of the linear heat equation, where the Maxwellian $N_{\gamma}$ is explicitly given by (2), in (9) the expression of the Maxwellian (8) is no more explicit in the physical space.

This situation has evident analogies with the central limit theorem of probability theory LR79. Indeed, while the Rosenau approximation of the heat equation is the analogue of the classical central limit theorem, and the large-time behavior is driven by a Gaussian density (the self-similar solution to the heat equation), the approximation (9) is the analogue of the central limit theorem for stable laws, where the expression of the stable law is explicitly known only in the Fourier variable.

Thanks to this analogy, the main features of the Maxwellian function (8) can be extracted from classical results on the central limit theorem for stable laws LR79, AA93, KOH95. The distribution function associated to the Maxwellian $M_{\lambda}$ belongs in fact to the domain of normal attraction of the Lévy symmetric stable distribution of order $\lambda$, defined by

$$
\widehat{L}_{\lambda}(\xi)=\mathrm{e}^{-|\xi|^{\lambda}} .
$$

We recall [Fel71, LR79 that a distribution function $F$ belongs to the domain of normal attraction of the stable law $L_{\lambda}(v) \mathrm{d} v$ if for any sequence of independent and identically distributed real-valued random variables $\left(X_{n}\right)_{n \geq 1}$ with common distribution function $F$ there exists a sequence of real numbers $\left(c_{n}\right)_{n \geq 1}$ such that the law of

$$
\frac{X_{1}+\cdots+X_{n}}{n^{1 / \lambda}}-c_{n}
$$

converges weakly to the stable law $L_{\lambda}(v) \mathrm{d} v$. We will come back to this and other properties of the Linnik distributions in Section 4 where we give a different characterization of the domain of normal attraction in terms of the asymptotic behavior at infinity. Without loss of generality, we will restrict our analysis to centered distributions, so that $c_{n}=0$.

It is important to outline that the fractional diffusion equation (4) has a fundamental solution, given by the Lévy distribution of order $\lambda$. Indeed, the Fourier version (6) is easily solved to give the solution

$$
\widehat{g}(\xi, t)=\widehat{g}_{0}(\xi) \mathrm{e}^{-|\xi|^{\lambda} t} .
$$

In addition to the aforementioned difficulties related to the fact that both Linnik distributions and the Lévy distribution are not explicitly expressed in the physical space, a second main difference with respect to the case of the heat equation is that in this case the Maxwellian function has moments bounded only up to a certain order. These facts mean that it is not immediate to assert that the 
correction (7), for a fixed time and a sufficiently small $\varepsilon$, is a good approximation to the fractional diffusion equation. Moreover, it is not clear whether or not the large-time behavior of the solution to this approximation agrees with the large-time behavior of the solution to the fractional diffusion.

The aim of this article is to give an answer to the previous questions in the range $1<\lambda<2$ of fractional diffusion. Like in the case of the heat equation, these results underline that the Rosenautype approximation can be viewed as a particular case of a general approximation to the fractional diffusion equation by means of a linear kinetic equation of type (9), provided the background density $M_{\lambda}$ is a probability density function which lies in the domain of normal attraction of the stable law (10). In Theorem 4, it will be shown that, in a certain metric equivalent to the weak*-convergence of measures, the distance between the solution to the fractional diffusion equation and the solution to the kinetic equation can be bounded in terms of $\varepsilon$ and $t$. While this bound allows us to obtain convergence for $\varepsilon \rightarrow 0$, validating in this way the approximating model, the same bound is not enough to get convergence for large times, even in this weak sense. The solution of equation (9), which in the Fourier variable can be easily written as

$$
\widehat{g}_{\varepsilon}(\xi, t)=\mathrm{e}^{-t \varepsilon^{-\lambda}\left(1-\widehat{M}_{\varepsilon, \lambda}(\xi)\right)} \widehat{g}_{0}(\xi)
$$

can be expressed at least in a formal way, as a convolution in the physical space

$$
g_{\varepsilon}(v, t)=P_{\lambda, \varepsilon}(\cdot, t) * g_{0}(v) .
$$

At difference with the solution to the fractional diffusion equation (11), since $\widehat{M}_{\lambda, \varepsilon} \in C_{0}(\mathbb{R})$ it follows that $P_{\lambda, \varepsilon}(v, t)$ cannot belong to $L^{1}(\mathbb{R})$ at any positive time. Indeed, as we will see in Section 3 resorting to a suitable expansion

$$
P_{\lambda, \varepsilon}(v, t)=\mathrm{e}^{-\varepsilon^{-\lambda} t} \delta_{0}+\mathrm{e}^{-\varepsilon^{-\lambda}} \sum_{n=1}^{\infty}\left(\frac{t}{\varepsilon^{\lambda}}\right)^{n} \frac{1}{n !} M_{\lambda, \varepsilon}^{* n}(v) .
$$

one identifies in the above expression a singular part $\mathrm{e}^{-\varepsilon^{-\lambda} t} \delta_{0}$, which vanishes exponentially fast both for $\varepsilon \rightarrow 0$ and $t \rightarrow+\infty$, and a regular part belonging to $L^{1}(\mathbb{R})$. For the particular case when $M_{\lambda}$ is a Linnik distribution (8), we will be able to recover the long time asymptotic behavior in $L^{1}(\mathbb{R})$ at the price of discarding this singular part. This will be done by introducing a regularized approximated solution, in which the convolution kernel $P_{\lambda, \varepsilon}$ is replaced by

$$
P_{\lambda, \varepsilon, r e g}(v, t)=P_{\lambda, \varepsilon}(v, t)+\mathrm{e}^{-\varepsilon^{-\lambda} t}\left(M_{\lambda, \varepsilon}(v)-\delta_{0}(v)\right)
$$

obtained by substituting the $\mathrm{e}^{-\varepsilon^{-\lambda} t} \delta_{0} * g_{0}$ term by $\mathrm{e}^{-\varepsilon^{-\lambda} t} M_{\lambda, \varepsilon} * g_{0}$.

The plan on the article is then as follows. In Section 2 we list various properties of the fractional diffusion equation. In particular, we prove convergence in $L^{1}(\mathbb{R})$ for large times to the fundamental solution (11). In Section 3, using tools of the kinetic theory of rarefied gases, we introduce a possible derivation and the main features of the Rosenau approximation with a general Maxwellian belonging to the domain of normal attraction of the stable law (10). This kinetic formulation is used to obtain explicit solutions to the Rosenau equation (9), using both Fourier transform and Wild sums [PT13. In Section 4 we deal with the problem of approximating the fractional diffusion at any fixed finite time with the Rosenau-type equation (9) with a general Maxwellian. Last, in Section 5, we investigate the large time behavior of the solutions to (9) with a Linnik distribution as a Maxwellian. We first show that with a suitable time-scaling, the approximated solution behaves asymptotically in time as the solution of the fractional diffusion equation in a suitable Fourier-based metric (see next subsection). As a consequence, both scaled solutions converge in the same metric towards the asymptotic profile of the fundamental solution. Then, we show that after a suitable regularization of the Rosenau equation, obtained by discarding its singular part, the approximated solution behaves asymptotically in $L^{1}(\mathbb{R})$ as the solution of the fractional diffusion equation.

The case $\lambda=1$ is a special case, since $\bar{M}_{1}(\xi)=(1+|\xi|)^{-1}$ is not in $L^{1}(\mathbb{R})$ and we did not succeed in performing analogous calculations as in the cases $1<\lambda<2$. However, the same results can be 
obtained with minor modifications provided the Linnik distribution $\widehat{M}_{1}(\xi)$ is replaced in equation (9) by the Lévy distribution $\widehat{L}_{1}(\xi)=\mathrm{e}^{-|\xi|}$ itself.

These technical results possess an evident interest for a consistent numerical approximation of the fractional diffusion equation (44). Indeed, let us start with a probability density function $g(v, t=0)=$ $g_{0}(v)$. Then a semi-implicit Euler scheme applied to the kinetic equation (9) shows that, in a fixed small time-interval $\Delta t$, the solution can be approximated according to the rule

$$
g(v, t+\Delta t)=\frac{\varepsilon}{\varepsilon+\Delta t} g(v, t)+\frac{\Delta t}{\varepsilon+\Delta t} M_{\lambda, \varepsilon} * g(v, t) .
$$

Therefore, at the time step $n+1$, the solution is obtained by a convex combination of the solution at the time step $n$, and of the convolution between the solution at the time step $n$ with the constant Maxwellian $M_{\lambda, \varepsilon}$. In particular, expression (12) can be easily implemented by Monte Carlo methods PT13.

\subsection{Functional framework}

Before entering into the main topic of this paper, we list below the various functional spaces, distances and norms used in our analysis. For $p \in[1,+\infty)$ and $q \in[1,+\infty)$, we denote by $L_{q}^{p}$ the weighted Lebesgue spaces

$$
L_{q}^{p}(\mathbb{R}):=\left\{f: \mathbb{R} \rightarrow \mathbb{R} \text { measurable; }\|f\|_{L_{q}^{p}}^{p}:=\int_{\mathbb{R}}|f(v)|^{p}\left(1+v^{2}\right)^{q / 2} \mathrm{~d} v<+\infty\right\} .
$$

In particular, the usual Lebesgue spaces are given by

$$
L^{p}:=L_{0}^{p}
$$

Moreover, for $f \in L_{q}^{1}$, we can define for any $\alpha \leq q$ the $\alpha^{\text {th }}$ order moment of $f$ as the quantity

$$
m_{\alpha}(f):=\int_{\mathbb{R}} f(v)|v|^{\alpha} \mathrm{d} v<+\infty .
$$

For $s \in \mathbb{N}$, we denote by $W^{s, p}$ the Sobolev spaces

$$
W^{s, p}(\mathbb{R}):=\left\{f \in L^{s} ;\|f\|_{W s, p}^{p}:=\sum_{|k| \leq s} \int_{\mathbb{R}}\left|f^{(k)}(v)\right|^{p} \mathrm{~d} v<+\infty\right\} .
$$

If $p=2$ we set $H^{s}:=W^{s, 2}$.

Given a probability density $f$, we define its Fourier transform $\mathcal{F}_{v}(f)$ by

$$
\mathcal{F}_{v}(f)(\xi)=\widehat{f}(\xi):=\int_{\mathbb{R}} f(v) e^{-i \xi v} \mathrm{~d} v, \quad \xi \in \mathbb{R}
$$

and the inverse Fourier transform as

$$
\varphi^{\vee}(v)=\frac{1}{2 \pi} \int_{\mathbb{R}} \varphi(\xi) \mathrm{e}^{i \xi v} \mathrm{~d} \xi .
$$

The Sobolev space $H^{s}$ can equivalently be defined for any $s \geq 0$ by the norm

$$
\|f\|_{H^{s}}:=\left\|\mathcal{F}_{v}(f)\right\|_{L_{2 s}^{2}} .
$$

The homogeneous Sobolev space $\dot{H}^{s}$ is then defined by the homogeneous norm

$$
\|f\|_{\dot{H}^{s}}^{2}:=\int_{\mathbb{R}}|\xi|^{2 s}|\widehat{f}(\xi)|^{2} \mathrm{~d} \xi .
$$


Finally, we introduce a family of Fourier-based metrics in the following way: given $s>0$ and two probability densities $f$ and $g$, their Fourier-based distance $d_{s}(f, g)$ is the quantity

$$
d_{s}(f, g):=\sup _{\xi \neq 0} \frac{|\widehat{f}(\xi)-\widehat{g}(\xi)|}{|\xi|^{s}} .
$$

This distance is finite, provided that $f$ and $g$ have finite moment of order $s$ and

$$
\int v^{k} f(v) \mathrm{d} v=\int v^{k} g(v) \mathrm{d} v, \quad k=1,2, \ldots,[s]
$$

where, if $s \notin \mathbb{N},[s]$ denotes the entire part of $s$ (or up to order $s-1$ if $s \in \mathbb{N}$ ). Moreover $d_{s}$ is an ideal metric [CT07. Its main properties are the following

i) For all probability densities $f, g, h$,

$$
d_{s}(f * h, g * h) \leq d_{s}(f, g) ;
$$

ii) Define for a given nonnegative constant $a$ the dilation

$$
f_{a}(v)=\frac{1}{a} f\left(\frac{v}{a}\right) .
$$

Then for any pair of probability densities $f, g$, and any nonnegative constant $a$

$$
d_{s}\left(f_{a}, g_{a}\right)=a^{s} d_{s}(f, g) .
$$

The $d_{s}$-metric is related to other more known metrics of large use in probability theory GTW95. In particular, two classical interpolation inequalities (see CGT99] for proofs) will be used in the following:

$$
\begin{aligned}
& \|f\|_{L^{1}} \leq C\|f\|_{L^{2}}^{\frac{2 \alpha}{1+2 \alpha}} m_{\alpha}(f)^{\frac{1}{1+2 \alpha}}, \quad \alpha>0 \\
& \|f\|_{L^{2}} \leq C\left(\sup _{\xi \neq 0} \frac{|\widehat{f}(\xi)|}{|\xi|^{s}}\right)^{\frac{2 s}{1+4 s}}\|f\|_{H^{s}}^{\frac{1+2 s}{1+4 s}}, \quad s>0 .
\end{aligned}
$$

\section{The fractional diffusion equation}

We recall here the existence result for the Cauchy problem associated to the fractional diffusion equation of order $\lambda, 0<\lambda<2$, with initial data $g_{0} \in L^{1}(\mathbb{R})$,

$$
\left\{\begin{array}{l}
\partial_{t} g(v, t)=-(\sqrt{-\Delta})^{\lambda} g(v, t), \quad v \in \mathbb{R}, t>0 \\
g(v, 0)=g_{0}(v)
\end{array}\right.
$$

and we briefly list properties of the solution that are used in our analysis.

By considering equation (15) in the Fourier variable it is straightforward to show that for any initial data $g_{0} \in L^{1}$ there exists a unique solution

$$
g(v, t)=\frac{1}{t^{1 / \lambda}} L_{\lambda}\left(\frac{\cdot}{t^{1 / \lambda}}\right) * g_{0}(v)
$$

where $L_{\lambda}$ is the Lévy distribution of order $\lambda$ defined by $\widehat{L}_{\lambda}(\xi)=\mathrm{e}^{-|\xi|^{\lambda}}$. For the sake of simplicity we denote the fundamental solution of the fractional diffusion equation by

$$
P_{\lambda}(v, t)=\frac{1}{t^{1 / \lambda}} L_{\lambda}\left(\frac{v}{t^{1 / \lambda}}\right)
$$


and so

$$
g(v, t)=P_{\lambda}(\cdot, t) * g_{0}(v) .
$$

To outline the analogies between the present problem and the classical central limit theorem for stable laws [LR79], we will further assume in the rest of the paper that $g_{0}$ is a probability density function. By mass conservation, the solution $g(t)$ will remain a probability density for all subsequent times.

It is well known [LR79] that in the interval $0<\lambda<2, L_{\lambda}$ belongs to $C_{0}(\mathbb{R})$ and it is an even probability density. For $\lambda=1$ the Lévy symmetric stable distribution coincides with the Cauchy distribution

$$
L_{1}(v)=\frac{1}{\pi} \frac{1}{1+v^{2}}
$$

Even though for $\lambda \neq 1 L_{\lambda}(v)$ is not known explicitly in the physical variable, its behavior for large $v$ is known in details Pól23. It holds

$$
L_{\lambda}(v) \sim \frac{1}{\pi} \Gamma(1+\lambda) \sin \left(\frac{\pi \lambda}{2}\right)|v|^{-(1+\lambda)}, \quad|v| \rightarrow+\infty .
$$

In consequence the heavy tailed density $L_{\lambda}$ has bounded moments only up to some order

$$
\int_{\mathbb{R}}|v|^{\alpha} L_{\lambda}(v) \mathrm{d} v=m_{\alpha}\left(L_{\lambda}\right)<+\infty, \quad 0<\alpha<\lambda
$$

The case $\lambda=2$ in equation (15) corresponds to the heat equation

$$
\left\{\begin{array}{l}
\partial_{t} g(v, t)=\Delta g(v, t) \quad v \in \mathbb{R}, t>0 \\
g(v, 0)=g_{0}(v) \in L^{1}(\mathbb{R}) .
\end{array}\right.
$$

In this case it is well known that the explicit solution is

$$
g(v, t)=\Omega(\cdot, t) * g_{0}(v)
$$

where $\Omega(v, t)=\frac{1}{\sqrt{4 \pi t}} \mathrm{e}^{-\frac{v^{2}}{4 t}}$ is the heat kernel. It can be shown BBDE11, Tos96] that $g(t)$ behaves as the heat kernel as $t \rightarrow+\infty$, provided that $g_{0}$ is a probability density of finite energy and entropy, namely

$$
\int_{\mathbb{R}} v^{2} g_{0}(v) \mathrm{d} v<+\infty, \quad \int_{\mathbb{R}}\left|\ln g_{0}(v)\right| g_{0}(v) \mathrm{d} v<+\infty
$$

and more precisely the following bound is known to be sharp

$$
\|g(t)-\Omega(t)\|_{L^{1}} \leq \frac{C}{\sqrt{1+2 t}}, \quad t>0
$$

where $C$ is an explicit constant.

A similar behavior occurs for the solution of the fractional diffusion equation, but in this case it appears difficult to obtain an explicit rate of approximation. However one can state the following proposition.

\section{Proposition 1}

Let $g(t)$ be the solution of the Cauchy problem (15), corresponding to the initial value $g_{0}$, a probability density function. Then

$$
\lim _{t \rightarrow+\infty}\left\|g(t)-\frac{1}{t^{1 / \lambda}} L_{\lambda}\left(\frac{\cdot}{t^{1 / \lambda}}\right)\right\|_{L^{1}}=0 .
$$


Proof: As in the case of the heat equation, this convergence can be obtained by passing from the fractional diffusion equation (15) to the corresponding Fokker-Planck equation

$$
\partial_{t} u(v, t)=-(\sqrt{-\Delta})^{\lambda} u(v, t)+\frac{2}{\lambda} \partial_{v}(v u(v, t))
$$

through the change of unknown function

$$
u(v, t)=\mathrm{e}^{\frac{2}{\lambda} t} g\left(\mathrm{e}^{\frac{2}{\lambda} t} v, \frac{\mathrm{e}^{2 t}-1}{2}\right)
$$

or, in an equivalent way,

$$
g(w, \tau)=\frac{1}{(\sqrt{2 \tau+1})^{2 / \lambda}} u\left(\frac{w}{(\sqrt{2 \tau+1})^{2 / \lambda}}, \frac{\ln (2 \tau+1)}{2}\right) .
$$

If we write equations (15) and (18) in the Fourier variable

$$
\begin{aligned}
& \partial_{t} \widehat{g}(\xi, t)=-|\xi|^{\lambda} \widehat{g}(\xi, t) \\
& \partial_{t} \widehat{u}(\xi, t)=-|\xi|^{\lambda} \widehat{u}(\xi, t)-\frac{2}{\lambda} \xi \partial_{\xi} \widehat{u}(\xi, t)
\end{aligned}
$$

and the explicit expression of the Fourier transform of the solution of the fractional diffusion equation with $g_{0}$ as initial data as $\widehat{g}(\xi, t)=\mathrm{e}^{-|\xi|^{\lambda} t} \widehat{g}_{0}(\xi)$, we get

$$
\widehat{u}(\xi, t)=\exp \left(-|\xi|^{\lambda} \frac{1-\mathrm{e}^{-2 t}}{2}\right) \widehat{g}_{0}\left(\mathrm{e}^{-\frac{2}{\lambda} t} \xi\right) .
$$

In [FPTT12, Proposition 3, it was proved that for a given probability density $g_{0}$ we have

$$
\lim _{t \rightarrow+\infty}\left\|u(t)-\mathcal{F}^{-1}\left(\exp \left(-\frac{|\xi|^{\lambda}}{2}\right)\right)\right\|_{L^{1}}=0 .
$$

This implies

$$
\lim _{\tau \rightarrow+\infty}\left\|u\left(\cdot, \frac{\ln (2 \tau+1)}{2}\right)-\mathcal{F}^{-1}\left(\exp \left(-\frac{|\xi|^{\lambda}}{2}\right)\right)\right\|_{L^{1}}=0 .
$$

By the scaling invariance of the $L^{1}$ norm we get

$$
\lim _{\tau \rightarrow+\infty} \| \frac{1}{(\sqrt{2 \tau+1})^{2 / \lambda}} u\left(\frac{\cdot}{(\sqrt{2 \tau+1})^{2 / \lambda}}, \frac{\ln (2 \tau+1)}{2}\right)-\mathcal{F}^{-1}\left(\exp \left(-\frac{\left|(\sqrt{2 \tau+1})^{2 / \lambda} \xi\right|^{\lambda}}{2}\right) \|_{L^{1}}=0 .\right.
$$

Therefore

$$
\lim _{t \rightarrow+\infty}\left\|g(t)-\mathcal{F}^{-1}\left(\exp \left(-\frac{(2 t+1)}{2}|\xi|^{\lambda}\right)\right)\right\|_{L^{1}}=0 .
$$

In order to get the desired result, it is enough to verify that

$$
\lim _{t \rightarrow+\infty}\left\|g(t)-\frac{1}{t^{1 / \lambda}} L_{\lambda}\left(\frac{v}{t^{1 / \lambda}}\right)\right\|_{L^{1}}=\lim _{t \rightarrow+\infty}\left\|g(t)-\mathcal{F}^{-1}\left(\mathrm{e}^{-t|\xi|^{\lambda}}\right)\right\|_{L^{1}}=0 .
$$

This follows since

$$
\lim _{t \rightarrow+\infty}\left\|\mathcal{F}^{-1}\left(\mathrm{e}^{-t|\xi|^{\lambda}}\right)-\mathcal{F}^{-1}\left(\exp \left(-\frac{(2 t+1)}{2}|\xi|^{\lambda}\right)\right)\right\|_{L^{1}}=0 .
$$


Indeed

$$
\begin{aligned}
& \mathcal{F}^{-1}\left(\mathrm{e}^{-t|\xi|^{\lambda}}\right)=\frac{1}{t^{1 / \lambda}} L_{\lambda}\left(\frac{v}{t^{1 / \lambda}}\right) \\
& \mathcal{F}^{-1}\left(\exp \left(-\frac{(2 t+1)}{2}|\xi|^{\lambda}\right)\right)=\frac{1}{(t+1 / 2)^{1 / \lambda}} L_{\lambda}\left(\frac{v}{(t+1 / 2)^{1 / \lambda}}\right)
\end{aligned}
$$

with $L_{\lambda}$ the Lévy distribution, with $L_{\lambda}(v) \leq \frac{C_{\lambda}}{1+|v|^{\lambda+1}}$ for all $v \in \mathbb{R}$.

Another important argument concerned with the solution of the fractional diffusion equation is the evolution of moments which are initially bounded. We already noticed in (17) that the moments $m_{\alpha}\left(L_{\lambda}\right)$ of $L_{\lambda}$ are bounded for $0<\alpha<\lambda$. Let us consider the Cauchy problem (15) with initial data $g_{0}$, a probability density with bounded moments in the range $0<\alpha<\lambda$,

$$
\int_{\mathbb{R}}|v|^{\alpha} g_{0}(v) \mathrm{d} v=m_{\alpha}\left(g_{0}\right)<+\infty .
$$

Since

and

$$
g(v, t)=P_{\lambda}(\cdot, t) * g_{0}(v)=\frac{1}{t^{1 / \lambda}} L_{\lambda}\left(\frac{\cdot}{t^{1 / \lambda}}\right) * g_{0}(v)
$$

$$
\int_{\mathbb{R}}|v|^{\alpha} P_{\lambda}(v, t) \mathrm{d} v=\int_{\mathbb{R}}|v|^{\alpha} \frac{1}{t^{1 / \lambda}} L_{\lambda}\left(\frac{v}{t^{1 / \lambda}}\right) \mathrm{d} v=t^{\alpha / \lambda} m_{\alpha}\left(L_{\lambda}\right)
$$

one obtains

$$
\begin{aligned}
& \int_{\mathbb{R}}|v|^{\alpha} P_{\lambda}(\cdot, t) * g_{0}(v) \mathrm{d} v=\int_{\mathbb{R}} \int_{\mathbb{R}}|v|^{\alpha} P_{\lambda}(v-w, t) g_{0}(w) \mathrm{d} w \mathrm{~d} v \\
& =\int_{\mathbb{R}} \int_{\mathbb{R}}|v-w+w|^{\alpha} P_{\lambda}(v-w, t) g_{0}(w) \mathrm{d} w \mathrm{~d} v .
\end{aligned}
$$

Let $0<\alpha \leq 1$. Then we get

$$
\begin{aligned}
& \int_{\mathbb{R}} \int_{\mathbb{R}}|v-w+w|^{\alpha} P_{\lambda}(v-w, t) g_{0}(w) \mathrm{d} w \mathrm{~d} v \leq \int_{\mathbb{R}} \int_{\mathbb{R}}\left(|v-w|^{\alpha}+|w|^{\alpha}\right) P_{\lambda}(v-w, t) g_{0}(w) \mathrm{d} w \mathrm{~d} v \\
& =t^{\alpha / \lambda} m_{\alpha}\left(L_{\lambda}\right)+m_{\alpha}\left(g_{0}\right) .
\end{aligned}
$$

If $1<\alpha<\lambda$

$$
\begin{aligned}
& \int_{\mathbb{R}} \int_{\mathbb{R}}|v-w+w|^{\alpha} P_{\lambda}(v-w, t) g_{0}(w) \mathrm{d} w \mathrm{~d} v \leq 2^{\alpha-1} \int_{\mathbb{R}} \int_{\mathbb{R}}\left(|v-w|^{\alpha}+|w|^{\alpha}\right) P_{\lambda}(v-w, t) g_{0}(w) \mathrm{d} w \mathrm{~d} v \\
& =2^{\alpha-1}\left(t^{\alpha / \lambda} m_{\alpha}\left(L_{\lambda}\right)+m_{\alpha}\left(g_{0}\right)\right) .
\end{aligned}
$$

In both cases, the moments of the solution are uniformly bounded above by an explicit function of time which grows as $t^{\lambda / \alpha}$.

We end this Section by proving that, in complete analogy with the heat equation, any convex functional is non-increasing along the solution to the fractional diffusion equation. First of all, we remark that for $t_{2}>t_{1}>0$

$$
\widehat{P}_{\lambda}\left(\xi, t_{2}\right)=\widehat{L}_{\lambda}\left(\xi t_{2}^{1 / \lambda}\right)=\mathrm{e}^{-|\xi|^{\lambda} t_{2}}=\mathrm{e}^{-|\xi|^{\lambda}\left(t_{2}-t_{1}\right)} \mathrm{e}^{-|\xi|^{\lambda} t_{1}}=\widehat{P}_{\lambda}\left(\xi, t_{2}-t_{1}\right) \widehat{P}_{\lambda}\left(\xi, t_{1}\right) .
$$

Owing to expression (16) for the solution we obtain, for $t_{2}>t_{1}>0$,

$$
g\left(v, t_{2}\right)=P_{\lambda}\left(\cdot, t_{2}\right) * g_{0}(v)=P_{\lambda}\left(\cdot, t_{2}-t_{1}\right) * g\left(\cdot, t_{1}\right)(v) .
$$

Now, let $\varphi(r), r \geq 0$ be a (smooth) convex function of $r$ and consider the functional

$$
\Phi(g)(t)=\int_{\mathbb{R}} \varphi(g(v, t)) \mathrm{d} v .
$$


If $t_{2}>t_{1}>0$, we get

$$
\Phi(g)\left(t_{2}\right)=\int_{\mathbb{R}} \varphi\left(g\left(v, t_{2}\right)\right) \mathrm{d} v=\int_{\mathbb{R}} \varphi\left(\int_{\mathbb{R}} P_{\lambda}\left(w, t_{2}-t_{1}\right) g\left(v-w, t_{1}\right) \mathrm{d} w\right) \mathrm{d} v .
$$

Now, use the fact that $P_{\lambda}$ is a probability density, so that by Jensen's inequality

$$
\begin{aligned}
\int_{\mathbb{R}} \varphi\left(\int_{\mathbb{R}} g\left(v-w, t_{1}\right) P_{\lambda}\left(w, t_{2}-t_{1}\right) \mathrm{d} w\right) \mathrm{d} v & \leq \iint_{\mathbb{R}^{2}} \varphi\left(g\left(v-w, t_{1}\right)\right) P_{\lambda}\left(w, t_{2}-t_{1}\right) \mathrm{d} v \mathrm{~d} w \\
& =\int_{\mathbb{R}} \varphi\left(g\left(v, t_{1}\right)\right) \mathrm{d} v=\Phi(g)\left(t_{1}\right) .
\end{aligned}
$$

Hence $\Phi(g)$ is non-increasing.

\section{The linear kinetic equation}

In this Section, we will briefly discuss both the derivation and the main properties of the linear kinetic equation (9). Let us consider a system composed of many identical particles. Let the number of particles with velocity $v$ at time $t$ be described by the process $X(t)$ with probability density $g(t)$, and suppose that the variation of $X(t)$ is solely due to interaction with an external background. The background $B_{\lambda}$ is here described by a random variable with probability density $M_{\lambda}$, which we will assume in the domain of attraction of a Lévy distribution of order $\lambda$, given by (10). Let us further assume that the interaction process of a particle with velocity $v$ with a background particle with velocity $w$ generates a post-interaction velocity $v^{*}$ given by

$$
v^{*}=v+w .
$$

In terms of the process $X(t)$ the law of change given by (24) can be rewritten as

$$
X^{*}(t)=X(t)+B_{\lambda}
$$

which implies, in the case in which $B_{\lambda}$ and $X(t)$ are independent, that the law of $X^{*}(t)$ is the convolution of the laws of $B_{\lambda}$ and $X(t)$. Assuming that $X(t), B_{\lambda}$ are independent each other, for a given observable quantity $\varphi(\cdot)$, we then have that the mean value of $\varphi(X)$ satisfies

$$
\frac{\mathrm{d}}{\mathrm{d} t} \int_{\mathbb{R}} \varphi(v) g(v, t) \mathrm{d} v=\frac{\mathrm{d}}{\mathrm{d} t}\langle\varphi(X(t))\rangle=\sigma \iint_{\mathbb{R} \times \mathbb{R}}\left(\varphi\left(v^{*}\right)-\varphi(v)\right) g(v, t) M_{\lambda}(w) \mathrm{d} v \mathrm{~d} w,
$$

where the constant $\sigma>0$ denotes as usual the interaction frequency. Note that choosing $\varphi(v)=1$ one shows that, independently of the background distribution, $g(t)$ remains a probability density if it so initially

$$
\int_{\mathbb{R}} g(v, t) \mathrm{d} v=\int_{\mathbb{R}} g_{0}(v) \mathrm{d} v=1 .
$$

This is in general the unique conservation law associated to equation (25).

The effects of the background can be easily modulated by considering, for a given small positive parameter $\varepsilon$, the random variable $\varepsilon B_{\lambda}$. To emphasize this dependence, we will denote its distribution as

$$
M_{\lambda, \varepsilon}(w)=\varepsilon^{-1} M_{\lambda}\left(\varepsilon^{-1} w\right) .
$$

Then, by inserting $M_{\lambda, \varepsilon}(w)$ into (25), and setting the interaction frequency $\sigma=1 / \varepsilon^{\lambda}$, the kinetic equation (25) coincides with the Rosenau approximation (9) in weak form. Hence, the Rosenau approximation of a fractional diffusion equation of order $\lambda$ describes a system of particles which modify their distribution through interactions with a background distributed according to a probability law in the domain of attraction of a Lévy stable law of order $\lambda$. 


\subsection{Representations of the solution to the Rosenau approximation}

The Rosenau approximated equation

$$
\partial_{t} g_{\varepsilon}(v, t)=\frac{1}{\varepsilon^{\lambda}}\left[M_{\lambda, \varepsilon} * g_{\varepsilon}(v, t)-g_{\varepsilon}(v, t)\right], \quad \varepsilon \ll 1
$$

where $\widehat{M}_{\lambda, \varepsilon}(\xi)=\widehat{M}_{\lambda}(\varepsilon \xi)$ and $M_{\lambda}$ belongs to the domain of normal attraction of the stable law (10), is a linear non local kinetic equation of Boltzmann type. Existence results for this equation are wellestablished. To find a solution of the Cauchy problem with $g_{0} \in L^{1}$ as initial data, we can resort to two equivalent methods. Resorting to the equation in the Fourier variable one can get a first explicit representation of the solution. This solution can be expressed as

$$
g_{\varepsilon}(v, t)=P_{\lambda, \varepsilon}(\cdot, t) * g_{0}(v),
$$

where, in the Fourier variable

$$
\widehat{P}_{\lambda, \varepsilon}(\xi, t)=\mathrm{e}^{-\varepsilon^{-\lambda} t\left(1-\widehat{M}_{\lambda, \varepsilon}(\xi)\right)} .
$$

We underline that, since $M_{\lambda} \in L^{1}(\mathbb{R})$ for every $t>0, \widehat{P}_{\lambda, \varepsilon}(\xi, t) \notin C_{0}(\mathbb{R})$ and consequently $P_{\lambda, \varepsilon}(v, t) \notin$ $L^{1}(\mathbb{R})$. This (unpleasant) feature of $P_{\lambda, \varepsilon}(\cdot, t)$ will appear in a clearer way by applying the so-called Wild sum expansion.

This expansion allows a useful representation of the solution of equation (26). It has been first introduced by Wild to construct a solution to the Boltzmann equation for Maxwell molecules [Wil51, and it appears well adapted to both linear and nonlinear kinetic equations PT13. Let $h_{\varepsilon}(v, t)=$ $\mathrm{e}^{\varepsilon^{-\lambda} t} g_{\varepsilon}(v, t)$. Then the Cauchy problem associated to equation (26) can be written as a fixed point problem as follows. Since

$$
\left\{\begin{array}{l}
\left(\partial_{t} g_{\varepsilon}(v, t)+\frac{1}{\varepsilon^{\lambda}} g_{\varepsilon}(v, t)\right) \mathrm{e}^{\varepsilon^{-\lambda} t}=\frac{\mathrm{e}^{\varepsilon^{-\lambda} t}}{\varepsilon^{\lambda}} M_{\lambda, \varepsilon} * g_{\varepsilon}(\cdot, t)(v) \\
g_{\varepsilon}(v, 0)=g_{0}(v),
\end{array}\right.
$$

then

$$
g_{\varepsilon}(v, t) \mathrm{e}^{\varepsilon^{-\lambda} t}-g_{\varepsilon}(v, 0)=\frac{1}{\varepsilon^{\lambda}} \int_{0}^{t} \mathrm{e}^{\varepsilon^{-\lambda} s} M_{\lambda, \varepsilon} * g_{\varepsilon}(v, s) \mathrm{d} s .
$$

Therefore

$$
h_{\varepsilon}(v, t)=g_{0}(v)+\frac{1}{\varepsilon^{\lambda}} \int_{0}^{t} M_{\lambda, \varepsilon} * h_{\varepsilon}(v, s) \mathrm{d} s=\Phi_{\varepsilon}\left(h_{\varepsilon}\right)(v, t) .
$$

Starting from

$$
h_{\varepsilon}^{(0)}(v)=g_{0}(v)
$$

and defining for any $n \geq 0$

$$
h_{\varepsilon}^{(n+1)}(v, t)=\Phi_{\varepsilon}\left(h_{\varepsilon}^{(n)}\right)(v, t)
$$

we construct the monotone sequence

$$
h_{\varepsilon}^{(n)}(v, t)=h_{\varepsilon}^{(n-1)}(v, t)+\left(t \varepsilon^{-\lambda}\right)^{n} \frac{1}{n !} M_{\lambda, \varepsilon}^{* n} * g_{0}(v),
$$

which converges in $L^{1}(\mathbb{R})$ towards

$$
h_{\varepsilon}(v, t)=g_{0}(v)+\sum_{n=1}^{\infty}\left(\frac{t}{\varepsilon^{\lambda}}\right)^{n} \frac{1}{n !} M_{\lambda, \varepsilon}^{* n} * g_{0}(v) .
$$

Finally, we obtain the expression

$$
g_{\varepsilon}(v, t)=\mathrm{e}^{-\varepsilon^{-\lambda} t} g_{0}(v)+\mathrm{e}^{-\varepsilon^{-\lambda} t} \sum_{n=1}^{\infty}\left(\frac{t}{\varepsilon^{\lambda}}\right)^{n} \frac{1}{n !} M_{\lambda, \varepsilon}^{* n} * g_{0}(v) .
$$


By comparing (29) with (27) one obtains an explicit representation of the fundamental solution $P_{\lambda, \varepsilon}(\cdot, t)$

$$
P_{\lambda, \varepsilon}(v, t)=\mathrm{e}^{-\varepsilon^{-\lambda}} t \delta_{0}(v)+\mathrm{e}^{-\varepsilon^{-\lambda} t} \sum_{n=1}^{\infty}\left(\frac{t}{\varepsilon^{\lambda}}\right)^{n} \frac{1}{n !} M_{\lambda, \varepsilon}^{* n}(v) .
$$

At difference with the fundamental solution of the original fractional diffusion equation (4), expression (30) shows that $P_{\lambda, \varepsilon}(\cdot, t)$ contains a singular part, the Dirac delta function $\delta_{0}$ located in $v=0$, of size exponentially decaying with time and $\varepsilon$.

\subsection{Properties of the solution to the Rosenau approximation}

Equation (25) allows us to control the time evolution of the moments of $g(t)$. For a given constant $\alpha>0$, let us take $\varphi(v)=|v|^{\alpha}$. We obtain

$$
\frac{\mathrm{d}}{\mathrm{d} t} \int_{\mathbb{R}}|v|^{\alpha} g(v, t) \mathrm{d} v=\frac{1}{\varepsilon^{\lambda}} \iint_{\mathbb{R}^{2}}\left[\left|v^{*}\right|^{\alpha}-|v|^{\alpha}\right] g(v, t) M_{\lambda, \varepsilon}(w) \mathrm{d} v \mathrm{~d} w .
$$

Since $\left|v^{*}\right|^{\alpha}=|v+w|^{\alpha} \leq c_{\alpha}\left(|v|^{\alpha}+|w|^{\alpha}\right)$, the moment of $g(t)$ of order $\alpha$ is bounded if the corresponding moment of the background distribution is bounded. Proceeding as in Lemma 11 one shows that the evolution of the moments is polynomial in time, in perfect agreement with the evolution of the corresponding moments for the solution of the fractional diffusion equation, as given by (22), (23) (see Remark 13).

Having in mind the discussion in RT13, a further interesting analogy with the linear diffusion is found by looking at the evolution of convex functionals along the solution. Let $\varphi(r), r \geq 0$ be a (smooth) convex function of $r$ and consider

$$
\Phi(g)(t)=\int_{\mathbb{R}} \varphi(g(v, t)) \mathrm{d} v .
$$

Then, using equation (9) we obtain

$$
\begin{gathered}
\frac{\mathrm{d}}{\mathrm{d} t} \Phi(g)(t)=\frac{\mathrm{d}}{\mathrm{d} t} \int_{\mathbb{R}} \varphi(g(v, t)) \mathrm{d} v=\int_{\mathbb{R}} \varphi^{\prime}(g(v, t)) \frac{\partial g(v, t)}{\partial t} \mathrm{~d} v= \\
\frac{1}{\varepsilon^{\lambda}} \int_{\mathbb{R}} \varphi^{\prime}(g(v, t))\left(M_{\lambda, \varepsilon} * g(v, t)-g(v, t)\right) \mathrm{d} v .
\end{gathered}
$$

Thanks to the convexity of $\varphi$, for $r, s \geq 0$

$$
\varphi^{\prime}(s)(r-s) \leq \varphi(r)-\varphi(s),
$$

and one obtains

$$
\frac{\mathrm{d}}{\mathrm{d} t} \int_{\mathbb{R}} \varphi(g(v, t)) \mathrm{d} v \leq \frac{1}{\varepsilon^{\lambda}} \int_{\mathbb{R}}\left(\varphi\left(M_{\lambda, \varepsilon} * g(v, t)\right)-\varphi(g(v, t))\right) \mathrm{d} v .
$$

Now, use the fact that $M_{\lambda, \varepsilon}$ is a probability density, so that by Jensen's inequality

to conclude

$$
\begin{gathered}
\int_{\mathbb{R}} \varphi\left(M_{\lambda, \varepsilon} * g(v, t)\right) \mathrm{d} v=\int_{\mathbb{R}} \varphi\left(\int_{\mathbb{R}} g(v-w, t) M_{\lambda, \varepsilon}(w) \mathrm{d} w\right) \mathrm{d} v \leq \\
\int_{\mathbb{R}^{2}} \varphi(g(v-w, t)) M_{\lambda, \varepsilon}(w) \mathrm{d} v \mathrm{~d} w=\int_{\mathbb{R}} \varphi(g(v, t)) \mathrm{d} v
\end{gathered}
$$

$$
\frac{\mathrm{d}}{\mathrm{d} t} \int_{\mathbb{R}} \varphi(g(v, t)) \mathrm{d} v \leq 0 .
$$

Thus any convex functional is non-increasing along the solution to the Rosenau type kinetic equation (9). 


\section{An approximation result}

As briefly discussed in the Introduction, one of the main novelties that can be extracted by the Rosenau approximation is that the kinetic model (26) has an evident interest from the point of view of its numerical approximation. This feature has been extensively investigated in the case of the linear diffusion in RT13, where it has been shown that the linear kinetic model represents a consistent approximation of the heat equation even if the Maxwellian density generated by the Rosenau idea is substituted by a different one, provided that some properties about moments are fulfilled. One of the results of this investigation has been the inclusion of a singular Maxwellian producing the central difference scheme, among the admissible Maxwellian densities for the corresponding linear kinetic model. Trying to get a similar result for the problem under consideration, we consider a linear kinetic equation of type (26)

$$
\partial_{t} g_{\varepsilon}(v, t)=\frac{1}{\varepsilon^{\lambda}}\left[M_{\lambda, \varepsilon} * g_{\varepsilon}(v, t)-g_{\varepsilon}(v, t)\right],
$$

in which the Linnik density (8) is replaced by a Maxwellian $M_{\lambda}$ with the properties to be even and the density of a centered distribution function belonging to the domain of normal attraction of the stable law $L_{\lambda}(v) \mathrm{d} v$, of exponent $\lambda \in(1,2)$. The evident advantage to work with a Maxwellian density different from Linnik distribution is that, as we will see, one can resort to a density which is explicitly known in the physical space.

In the rest of this section, we aim at proving that, with a suitable choice of distance, at any fixed time the solutions to (26) and to the fractional diffusion equation, are getting closer in terms of the parameter $\varepsilon$. First of all we underline that it is not even clear in which sense the solution to (26) represents an approximation to the solution of the fractional diffusion equation (4) as $\varepsilon$ tends to zero. This is in contrast with what happens to the original Rosenau approximation to the heat equation (3). In this case the Maxwellian function $N_{\varepsilon}(v)=\frac{1}{2 \varepsilon} \mathrm{e}^{-\frac{|v|}{\varepsilon}}$ has finite moments of any order, and the meaning of approximation is standard. Let us consider equation (3) in weak form

$$
\frac{\mathrm{d}}{\mathrm{d} t} \int_{\mathbb{R}} g_{\varepsilon}(v, t) \varphi(v) \mathrm{d} v=\frac{1}{\varepsilon^{2}} \iint_{\mathbb{R}^{2}}(\varphi(v+w)-\varphi(v)) g_{\varepsilon}(v, t) N_{\varepsilon}(w) \mathrm{d} v \mathrm{~d} w .
$$

By expanding $\varphi(v+w)$ in Taylor series around $v$

$$
\varphi(v+w)=\varphi(v)+\varphi^{\prime}(v) w+\frac{\varphi^{\prime \prime}(v)}{2} w^{2}+\frac{\varphi^{\prime \prime \prime}(\tilde{v})}{3 !} w^{3}, \quad \tilde{v} \in(v, w)
$$

we get

$$
\frac{\mathrm{d}}{\mathrm{d} t} \int_{\mathbb{R}} g_{\varepsilon}(v, t) \varphi(v) \mathrm{d} v=\frac{1}{\varepsilon^{2}} \iint_{\mathbb{R}^{2}}\left(\varphi^{\prime}(v) w+\frac{\varphi^{\prime \prime}(v)}{2} w^{2}+\frac{\varphi^{\prime \prime \prime}(\tilde{v})}{3 !} w^{3}\right) g_{\varepsilon}(v, t) N_{\varepsilon}(w) \mathrm{d} v \mathrm{~d} w .
$$

Since

$$
\int_{\mathbb{R}} w N_{\varepsilon}(w) \mathrm{d} w=0, \quad \int_{\mathbb{R}} w^{2} N_{\varepsilon}(w) \mathrm{d} w=2 \varepsilon^{2}, \quad \int_{\mathbb{R}}|w|^{3} N_{\varepsilon}(w) \mathrm{d} w=12 \varepsilon^{3},
$$

we end up with the equation

$$
\frac{\mathrm{d}}{\mathrm{d} t} \int_{\mathbb{R}} g_{\varepsilon}(v, t) \varphi(v) \mathrm{d} v=\int_{\mathbb{R}} g_{\varepsilon}(v, t) \varphi^{\prime \prime}(v) \mathrm{d} v+C(\varepsilon),
$$

where the remainder satisfies

$$
|C(\varepsilon)| \leq 2 \varepsilon\left\|\varphi^{\prime \prime \prime}\right\|_{L^{\infty}} \int_{\mathbb{R}}|w|^{3} N(w) \mathrm{d} w \rightarrow 0, \quad \varepsilon \rightarrow 0 .
$$

In the case of the fractional diffusion approximation, the operator $(\sqrt{-\Delta})^{\lambda}$ is non-local and the Maxwellian $M_{\lambda, \varepsilon}$ has finite moments only for $\alpha<\lambda$. This requires a different way of looking to 
the problem. We begin by defining in which sense we can consider equation (26) as an approximation of the fractional diffusion equation.

We recall that a centered distribution function $F$ belongs to the domain of normal attraction of the stable law $L_{\lambda}(v) \mathrm{d} v$ if for any sequence of independent and identically distributed real-valued random variables $\left(X_{n}\right)_{n \geq 1}$ with common distribution function $F$, the law of

$$
\frac{X_{1}+\cdots+X_{n}}{n^{1 / \lambda}}
$$

converges weakly to the stable law $L_{\lambda}(v) \mathrm{d} v$.

Let us recall some properties of a distribution $F$ belonging to the domain of normal attraction of a stable law. More information about this topic can be found in the book [L71] or, among others, in the papers [BLM11], BLR08. It is well-known that a centered distribution $F$ belongs to the domain of normal attraction of the $\lambda$-stable law (10) with density $L_{\lambda}(v)$ if and only if $F$ satisfies $|v|^{\lambda} F(v) \rightarrow c$ as $v \rightarrow-\infty$ and $v^{\lambda}(1-F(v)) \rightarrow c$ as $v \rightarrow+\infty$ i.e.

$$
\begin{aligned}
& F(-v)=\frac{c}{|v|^{\lambda}}+S_{1}(-v) \quad \text { and } \quad 1-F(v)=\frac{c}{v^{\lambda}}+S_{2}(v) \quad(v>0) \\
& S_{i}(v)=o\left(|v|^{-\lambda}\right) \quad \text { as }|v| \rightarrow+\infty, \quad i=1,2
\end{aligned}
$$

where $c=\frac{\Gamma(\lambda)}{\pi} \sin \left(\frac{\pi \lambda}{2}\right)$. Concerning the moments of the distribution function $F$ and of the distributions $F_{n}$ associated to the sum $\left(X_{1}+\cdots+X_{n}\right) / n^{1 / \lambda}$ considered in (31), we recall the following Propositions.

\section{Proposition 2 (see [L71], Theorem 2.6.4 page 84)}

Let $F$ belong to the domain of normal attraction of $L_{\lambda}$. Then, for any $\alpha$ such that $0<\alpha<\lambda$

$$
\int_{\mathbb{R}}|v|^{\alpha} \mathrm{d} F(v)<+\infty
$$

\section{Proposition 3 (see [L71, Lemma 5.2.2 page 142)}

Let $F_{n}$ denote the distribution function associated to the sum $\left(X_{1}+\cdots+X_{n}\right) / n^{1 / \lambda}$ converging weakly to the stable law $L_{\lambda}(v) \mathrm{d} v$. Then, for any $0<\alpha<\lambda$

$$
\int_{\mathbb{R}}|v|^{\alpha} \mathrm{d} F_{n}(v)
$$

is uniformly bounded with respect to $n$.

The behavior of $F$ in the physical space (32) leads to a characterization of the domain of normal attraction of $L_{\lambda}$ in terms of characteristic functions. Indeed, if $\Phi$ is the characteristic function of the distribution function $F$ satisfying (32) then

$$
1-\Phi(\xi)=\left(1+v_{0}(\xi)\right)|\xi|^{\lambda},
$$

where

$$
v_{0} \in L^{\infty}(\mathbb{R}) \quad \text { and } \quad\left|v_{0}(\xi)\right|=o(1), \quad|\xi| \rightarrow 0 .
$$

In the following we will consider a stronger assumption on the characteristic function. We will denote by $M_{\lambda}$ any density of a centered distribution function belonging to the domain of normal attraction of the stable law with density $L_{\lambda}$, which has the extra property that the Fourier-Stieltjes transform of the function $M_{\lambda}$ satisfies

$$
1-\widehat{M_{\lambda}}(\xi)=\left(1+v_{0}(\xi)\right)|\xi|^{\lambda},
$$

where $v_{0}(\xi)$ is such that, for some $\delta>0$

$$
v_{0} \in L^{\infty}(\mathbb{R}) \quad \text { and } \quad\left|v_{0}(\xi)\right|=O\left(|\xi|^{\delta}\right), \quad|\xi| \rightarrow 0 .
$$


A main example is furnished by the so-called Barenblatt function

$$
B_{\lambda}(v)=\frac{\alpha}{\left(1+(\beta v)^{2}\right)^{(1+\lambda) / 2}}, \quad v \in \mathbb{R}
$$

where $\alpha, \beta>0, \quad \frac{\beta}{\alpha}=\int_{\mathbb{R}} \frac{\mathrm{d} v}{\left(1+v^{2}\right)^{(1+\lambda) / 2}}$ and $\frac{\alpha}{\lambda \beta^{1+\lambda}}=\frac{\Gamma(\lambda) \sin \left(\frac{\pi \lambda}{2}\right)}{\pi}$. This type of functions is mainly related to the study of nonlinear equations for fast diffusion, given by

$$
\partial_{t} g(v, t)=\partial_{v v}^{2} g^{p}(v, t),
$$

as $p<1$. The Barenblatt function (35) corresponds to the case $p=(\lambda-1) /(\lambda+1)$. The function $t^{(\lambda+1) /(2 \lambda)} B_{\lambda}\left(t^{(\lambda+1) /(2 \lambda)} x\right)$ represents the intermediate asymptotic profile of the nonlinear diffusion (36) Vaz07. The case $p<1$ appears when modelling diffusion in metals and ceramic materials. In these materials, in fact, over a wide range of temperatures, the diffusion coefficient can be approximated as $u^{-\alpha}$, where $0<\alpha<2$ [Ros95].

The distribution function $F(x)=\int_{-\infty}^{x} B_{\lambda}(v) \mathrm{d} v$ is such that

$$
\lim _{x \rightarrow+\infty} x^{\lambda}(1-F(x))=\frac{\alpha}{\lambda \beta^{1+\lambda}}=\frac{\Gamma(\lambda) \sin \left(\frac{\pi \lambda}{2}\right)}{\pi}=c .
$$

Moreover for any $0<\delta<2$ there exists a constant $C>0$ such that for any $x>0$ we have

$$
\left|x^{\lambda+\delta}(1-F(x))-c x^{\delta}\right|<C .
$$

This in enough to guarantee that the Fourier transform of $B_{\lambda}$ satisfies the extra property (33)-(34) (see BLR08). Property (34) has been already considered in kinetic theory. In particular, it has been used to determine the rate of convergence to equilibrium for the dissipative Kac model [BLR08.

Under this condition on the Maxwellian function, we can prove convergence of the approximated solution (26) to the solution to the fractional diffusion equation (44) in the Fourier-based distance $d_{s}$, as $\varepsilon \rightarrow 0$.

\section{Theorem 4}

Let $1<\lambda<2$, and let $g(t)$ and $g_{\varepsilon}(t)$ be the solutions of the fractional diffusion equation (15) and, respectively, of the Rosenau approximation (26), corresponding to the same initial probability density $g_{0}$. Let us suppose moreover that the Maxwellian $M_{\lambda}$ in (26) satisfies the extra properties (33)- (34). Then for any $0<s<\lambda$ there exists a positive constant $C=C(\lambda, s, \delta)$ such that

$$
d_{s}\left(g(t), g_{\varepsilon}(t)\right) \leq C t^{s /(\lambda+\delta)} \varepsilon^{s \delta /(\lambda+\delta)} .
$$

Proof: Since $g_{0}$ is a probability density, $\left|\widehat{g}_{0}(\xi)\right| \leq 1$, and we have

$$
d_{s}\left(g(t), g_{\varepsilon}(t)\right)=\sup _{\xi \neq 0} \frac{\left|\widehat{g}_{0}(\xi)\left(\mathrm{e}^{-|\xi|^{\lambda} t}-\mathrm{e}^{-\varepsilon^{-\lambda} t\left(1-\widehat{M}_{\lambda, \varepsilon}(\xi)\right)}\right)\right|}{|\xi|^{s}} \leq \sup _{\xi \neq 0} \frac{\left|\mathrm{e}^{-|\xi|^{\lambda} t}-\mathrm{e}^{-\varepsilon^{-\lambda} t\left(1-\widehat{M}_{\lambda, \varepsilon}(\xi)\right)}\right|}{|\xi|^{s}} .
$$

Therefore, for any $R>0$

$$
d_{s}\left(g(t), g_{\varepsilon}(t)\right) \leq \frac{2}{R^{s}}+\sup _{0<|\xi| \leq R} \frac{\mid \mathrm{e}^{-|\xi|^{\lambda} t}-\mathrm{e}^{-\varepsilon^{-\lambda} t\left(1-\widehat{M}_{\lambda, \varepsilon}(\xi)\right) \mid}}{|\xi|^{s}} .
$$

Thanks to the inequality $\left|\mathrm{e}^{-x}-\mathrm{e}^{-y}\right| \leq|x-y|$, valid for any $x, y \geq 0$, we get

$$
\sup _{0<|\xi| \leq R} \frac{|\xi|^{\lambda} t-\varepsilon^{-\lambda} t\left(1-\widehat{M}_{\lambda, \varepsilon}(\xi)\right) \mid}{|\xi|^{s}} \leq \sup _{0<|\xi| \leq R} \frac{t|\xi|^{\lambda}\left|v_{0}(\varepsilon \xi)\right|}{|\xi|^{s}} .
$$


In the last inequality we used the expression of the Fourier transform of $M_{\lambda}$ (33). Thanks to (334) we obtain

$$
\sup _{0<|\xi| \leq R} \frac{t|\xi|^{\lambda}\left|v_{0}(\varepsilon \xi)\right|}{|\xi|^{s}} \leq C \sup _{0<|\xi| \leq R} \frac{t|\xi|^{\lambda}|\varepsilon \xi|^{\delta}}{|\xi|^{s}} \leq C t R^{\lambda+\delta-s} \varepsilon^{\delta} .
$$

Finally, choose $R=\left(\frac{2}{C t \varepsilon^{\delta}}\right)^{1 /(\lambda+\delta)}$ to obtain

$$
d_{s}\left(g(t), g_{\varepsilon}(t)\right) \leq C t^{s /(\lambda+\delta)} \varepsilon^{s \delta /(\lambda+\delta)}
$$

\section{$5 \quad$ Large time behavior}

The result of the previous section justifies the choice of a Barenblatt type Maxwellian density in the linear kinetic model (7), to obtain an explicit in space linear kinetic equation which approximates, at any fixed time, the fractional diffusion equation. The result of Theorem 4 , however, is such that the rate of convergence in the $d_{s}$-metric is time-dependent, and fails as $t \rightarrow \infty$. As the analysis in RT13. shows, the weakness of this result with respect to time could be generated by the choice of a general Maxwellian in the linear kinetic model, that, while maintaining the kinetic form of the approximation, is loosing the precise shape of the Maxwellian predicted by the Rosenau idea. Consequently, in this section we will restrict the study of the large time behavior of the solution to the approximated Rosenau equation (7), where $M_{\lambda, \varepsilon}$ is a Linnik distribution (8). Provided that we discard the singular part of the approximating solution, our analysis will confirm that in this case the solution to (7) behaves like the fractional diffusion equation for large times.

\subsection{Convergence in the Fourier based metric}

In analogy with the solution of the fractional diffusion equation, for all $\xi \in \mathbb{R}$ we have

$$
\lim _{t \rightarrow+\infty}\left(\widehat{g_{\varepsilon}}(t, \xi)-\mathrm{e}^{-|\xi|^{\lambda} t}\right)=0
$$

This convergence can be refined using the Fourier-based distance $d_{s}$. In order to capture the asymptotic profile in the limit for $t \rightarrow+\infty$ we consider the scaled solution obtained by the change of variable $\xi \longmapsto \frac{\xi}{(1+t)^{1 / \lambda}}$. Let us denote by

$$
\begin{aligned}
& h(v, t)=(1+t)^{1 / \lambda} g\left((1+t)^{1 / \lambda} v, t\right) \\
& h_{\varepsilon}(v, t)=(1+t)^{1 / \lambda} g_{\varepsilon}\left((1+t)^{1 / \lambda} v, t\right) .
\end{aligned}
$$

the scaled solutions of the fractional diffusion equation and of the approximated equation respectively. Then using the explicit representation of the solution established in (27)-(28), we get

$$
\begin{aligned}
\widehat{h}_{\varepsilon}(\xi, t) & =\widehat{P}_{\lambda, \varepsilon}\left(\frac{\xi}{(1+t)^{1 / \lambda}}, t\right) \widehat{g}_{0}\left(\frac{\xi}{(1+t)^{1 / \lambda}}\right) \\
& =\exp \left(-\varepsilon^{-\lambda} t\left(1-\widehat{M}_{\lambda, \varepsilon}\left(\frac{\xi}{(1+t)^{1 / \lambda}}\right)\right)\right) \widehat{g}_{0}\left(\frac{\xi}{(1+t)^{1 / \lambda}}\right)
\end{aligned}
$$

and since

$$
\varepsilon^{-\lambda} t\left(1-\widehat{M}_{\lambda, \varepsilon}\left(\frac{\xi}{(1+t)^{1 / \lambda}}\right)\right)=\frac{t|\xi|^{\lambda}}{1+t+\varepsilon^{\lambda}|\xi|^{\lambda}}
$$

for $t \rightarrow+\infty$ we get, for $\xi \in \mathbb{R}$ and $\varepsilon>0$ fixed

$$
\lim _{t \rightarrow+\infty} \widehat{h}_{\varepsilon}(\xi, t)=\exp \left(-|\xi|^{\lambda}\right) \widehat{g}_{0}(0) .
$$




\section{Proposition 5}

Let $1<\lambda<2$, and let $h(t)$ and $h_{\varepsilon}(t)$ be the solutions of (37), the scaled fractional diffusion equation (15) and, respectively, of its Rosenau approximation (7), corresponding to the same initial probability density $g_{0}$. If $d_{s}\left(g_{0}, L_{\lambda}\right)<+\infty$ for some $0<s<\lambda$, then there exists a positive constant $C=C(\lambda, s)$ such that

$$
d_{s}\left(h(t), h_{\varepsilon}(t)\right) \leq C \varepsilon^{s / 2} \frac{t^{s / 2 \lambda}}{(1+t)^{s / \lambda}},
$$

and

$$
d_{s}\left(h_{\varepsilon}(t), L_{\lambda}\right) \leq C\left(\frac{1}{(1+t)^{s / \lambda}}+\varepsilon^{s / 2} \frac{t^{s / 2 \lambda}}{(1+t)^{s / \lambda}}\right)
$$

Proof: By the scaling rule (13), we have

$$
d_{s}\left(h(t), h_{\varepsilon}(t)\right)=\frac{1}{(1+t)^{s / \lambda}} d_{s}\left(g(t), g_{\varepsilon}(t)\right) .
$$

The first inequality (38) follows therefore from Theorem 4 Indeed, $\widehat{M}_{\lambda}(\xi)=\frac{1}{1+|\xi|^{\lambda}}$ and so

$$
1-\widehat{M}_{\lambda}(\xi)=|\xi|^{\lambda}\left(1-\frac{|\xi|^{\lambda}}{1+|\xi|^{\lambda}}\right)=|\xi|^{\lambda}\left(1+v_{0}(\xi)\right)
$$

with

$$
v_{0}(\xi)=-\frac{|\xi|^{\lambda}}{1+|\xi|^{\lambda}}=O\left(|\xi|^{\lambda}\right), \quad|\xi| \rightarrow 0
$$

The second inequality (39) is a consequence of the first one. Indeed, we apply the triangular inequality

$$
d_{s}\left(h_{\varepsilon}(t), L_{\lambda}\right) \leq d_{s}\left(h_{\varepsilon}(t), h(t)\right)+d_{s}\left(h(t), L_{\lambda}\right),
$$

where

$$
d_{s}\left(h(t), L_{\lambda}\right)=\sup _{\xi \neq 0} \frac{\left|\widehat{g}_{0}\left(\frac{\xi}{(1+t)^{1 / \lambda}}\right) \exp \left(-\frac{|\xi|^{\lambda} t}{1+t}\right)-\exp \left(-|\xi|^{\lambda}\right)\right|}{|\xi|^{s}} \leq \frac{1}{(1+t)^{s / \lambda}} d_{s}\left(g_{0}, L_{\lambda}\right) .
$$

\subsection{Strong convergence in $L^{1}$ of a regularized solution}

As given by expression (30), the fundamental solution $P_{\lambda, \varepsilon}(\cdot, t)$ contains a singular part, with a size which is exponentially decaying to zero. The effect of this singular part on the solution to the approximation is clear. For this reason, we study here the large time behavior of the regularized part. The main result will be that this suitable regularized solution to the Rosenau approximation converges in strong sense (namely in $L^{1}$-norm) to the solution of the fractional diffusion equation.

We define the regularized fundamental solution to the Rosenau approximation by

$$
g_{\varepsilon, r e g}(v, t)=P_{\lambda, \varepsilon, r e g}(\cdot, t) * g_{0}(v)
$$

where

$$
P_{\lambda, \varepsilon, r e g}(v, t)=P_{\lambda, \varepsilon}(v, t)+\mathrm{e}^{-\varepsilon^{-\lambda} t}\left(M_{\lambda, \varepsilon}(v)-\delta_{0}(v)\right)
$$

\section{Remark 6}

Note that function (41) is obtained from (30) by substituting the singular part with the order zero term of the sum. In this way, the difference between the fundamental solution and the regularized one is still exponentially decaying to zero both with respect to time and $\varepsilon$, with the additional property to vanish at the point $\xi=0$. In other words, the regularized solution is constructed in such a way that the masses of $P_{\lambda, \varepsilon}(\cdot, t)$ and $P_{\lambda, \varepsilon, r e g}(\cdot, t)$ coincide. 


\section{Remark 7}

It is immediate to prove that $P_{\lambda, \varepsilon, r e g}(t) \in L^{1}(\mathbb{R})$ for each value of $t>0$. Indeed

$$
P_{\lambda, \varepsilon, r e g}(t)=\mathrm{e}^{-\varepsilon^{-\lambda} t} \sum_{n=1}^{+\infty}\left(t \varepsilon^{-\lambda}\right)^{n} \frac{1}{n !} M_{\lambda, \varepsilon}^{* n}+\mathrm{e}^{-\varepsilon^{-\lambda}} M_{\lambda, \varepsilon}
$$

and the Maxwellian term $M_{\lambda, \varepsilon}$ belongs to $L^{1}(\mathbb{R})$. Then the series in 42 ) converges in $L^{1}(\mathbb{R})$ for any $t>0$.

The main result of this paper is contained in the following

\section{Theorem 8}

Let $1<\lambda<2$, and let $g(t)$ and $g_{\varepsilon, r e g}(t)$ denote the solutions of the Cauchy problem for the fractional diffusion equation (15) and, respectively, the solution of the regularized Rosenau approximation (40), corresponding to the same initial density $g_{0}$. Then, if for all $0<\alpha<\lambda$, $g_{0}$ has bounded moment of $\operatorname{order} \alpha$

$$
\lim _{t \rightarrow+\infty}\left\|g_{\varepsilon, r e g}(t)-g(t)\right\|_{L^{1}}=0
$$

By Theorem 8 and Proposition 1 we get immediately the following Corollary.

\section{Corollary 9}

Let $1<\lambda<2$, and let $g_{0}$ be a probability density with bounded moment of order $\alpha$, for all $0<\alpha<\lambda$. Then

$$
\lim _{t \rightarrow+\infty}\left\|g_{\varepsilon, r e g}(t)-\frac{1}{t^{1 / \lambda}} L_{\lambda}\left(\frac{\cdot}{t^{1 / \lambda}}\right)\right\|_{L^{1}}=0
$$

with $L_{\lambda}$ the Lévy symmetric stable distribution (10).

The proof of Theorem 8 follows by various steps, we will split into different lemmas, that we will prove below. The first one is concerned with the convergence (after scaling) of the fundamental solution of the fractional diffusion equation to the regularized fundamental solution. For the sake of simplicity let us denote

$$
\begin{aligned}
& \tilde{P}_{\lambda}(v, t)=(1+t)^{1 / \lambda} P_{\lambda}\left((1+t)^{1 / \lambda} v, t\right), \\
& \tilde{P}_{\lambda, \varepsilon, r e g}(v, t)=(1+t)^{1 / \lambda} P_{\lambda, \varepsilon, r e g}\left((1+t)^{1 / \lambda} v, t\right) .
\end{aligned}
$$

The result follows in consequence of Proposition 5 .

\section{Lemma 10}

Let $1<\lambda<2$. For any $0<s<\lambda$ there exists a positive constant $C=C(\lambda, s)$ such that

$$
d_{s}\left(\tilde{P}_{\lambda}(t), \tilde{P}_{\lambda, \varepsilon, r e g}(t)\right) \leq C \frac{\varepsilon^{s / 2}}{(1+t)^{s /(2 \lambda)}}, \quad t>0 .
$$

The second lemma describes the growth of the $\alpha$-moment of the scaled fundamental solution of the fractional diffusion equation and of the scaled regularized fundamental solution of the Rosenau approximation.

\section{Lemma 11}

Let $1<\lambda<2$. For any $0<\alpha<\lambda$ and any $t>0$

$$
m_{\alpha}\left(\tilde{P}_{\lambda}(t)\right)=\int_{\mathbb{R}}|v|^{\alpha} \tilde{P}_{\lambda}(v, t) \mathrm{d} v \leq m_{\alpha}\left(L_{\lambda}\right) .
$$

Moreover, there exist positive constants $C=C(\lambda, \alpha)$ such that

$$
m_{\alpha}\left(\tilde{P}_{\lambda, \varepsilon, r e g}(t)\right)=\int_{\mathbb{R}}|v|^{\alpha} \tilde{P}_{\lambda, \varepsilon, r e g}(v, t) \mathrm{d} v \leq C .
$$


The third lemma deals with the evolution of the Sobolev norms of both the scaled fundamental solution of the fractional diffusion equation and of the scaled regularized fundamental solution of the Rosenau approximation.

\section{Lemma 12}

Let $1<\lambda<2$. For any $0<s<(\lambda-1) / 2$, any $0<\beta<1 / \lambda$ and for $t$ large enough there are positive constants $C_{1}=C_{1}(\lambda, s)$ and $C_{2}=C_{2}(\lambda, \beta, s)$ such that

$$
\begin{aligned}
& \left\|\tilde{P}_{\lambda}(t)\right\|_{\dot{H}^{s}} \leq C_{1}, \\
& \left\|\tilde{P}_{\lambda, \varepsilon, r e g}(t)\right\|_{\dot{H}^{s}} \leq C_{2} \frac{1}{\varepsilon^{s+1 / 2}}(1+t)^{(s+1 / 2)(1 / \lambda-\beta)} .
\end{aligned}
$$

With these results at hand, we can prove Theorem 8

Proof of Theorem 8. The proof of the theorem can be divided into different steps. Recalling that $\left\|g_{0}\right\|_{L^{1}}=1, g(t)=P_{\lambda}(t) * g_{0}$ and $g_{\varepsilon, r e g}(t)=P_{\lambda, \varepsilon, r e g}(t) * g_{0}$, we get for $t>0$

$$
\left\|g(t)-g_{\varepsilon, r e g}(t)\right\|_{L^{1}} \leq\left\|P_{\lambda}(t)-P_{\lambda, \varepsilon, r e g}(t)\right\|_{L^{1}} .
$$

Thanks to the invariance by scaling of the $L^{1}$ norm we get

$$
\left\|P_{\lambda}(t)-P_{\lambda, \varepsilon, r e g}(t)\right\|_{L^{1}}=\left\|\tilde{P}_{\lambda}(t)-\tilde{P}_{\lambda, \varepsilon, r e g}(t)\right\|_{L^{1}},
$$

with $\tilde{P}_{\lambda}$ and $\tilde{P}_{\lambda, \varepsilon, \text { reg }}$ defined in (43). By using the interpolation inequalities (14), for $t>0$ and $\alpha, s \in(0, \lambda)$ we obtain

$$
\begin{aligned}
&\left\|\tilde{P}_{\lambda}(t)-\tilde{P}_{\lambda, \varepsilon, r e g}(t)\right\|_{L^{1}} \leq C\left\|\tilde{P}_{\lambda}(t)-\tilde{P}_{\lambda, \varepsilon, r e g}(t)\right\|_{L^{2}}^{\frac{2 \alpha}{1+2 \alpha}}\left[m_{\alpha}\left(\tilde{P}_{\lambda}(t)\right)+m_{\alpha}\left(\tilde{P}_{\lambda, \varepsilon, r e g}(t)\right)\right]^{\frac{1}{1+2 \alpha}} \\
& \leq C d_{s}\left(\tilde{P}_{\lambda}(t), \tilde{P}_{\lambda, \varepsilon, r e g}(t)\right)^{\frac{4 s \alpha}{(1+4 s)(1+2 \alpha)}}\left\|\tilde{P}_{\lambda}(t)-\tilde{P}_{\lambda, \varepsilon, r e g}(t)\right\|_{\dot{H}^{s}}^{\frac{2 \alpha(1+2 s)}{(1+4 s)(1+2 \alpha)}} \\
& \\
& {\left[m_{\alpha}\left(\tilde{P}_{\lambda}(t)\right)+m_{\alpha}\left(\tilde{P}_{\lambda, \varepsilon, r e g}(t)\right)\right]^{\frac{1}{1+2 \alpha}} . }
\end{aligned}
$$

Thanks to estimate (44), the bounds in Lemmas 10, 11] and 12 imply

$$
\begin{aligned}
& \left\|\tilde{P}_{\lambda}(t)-\tilde{P}_{\lambda, \varepsilon, r e g}(t)\right\|_{L^{1}} \\
& \leq C\left[\frac{\varepsilon^{\frac{s}{2}}}{(1+t)^{\frac{s}{2 \lambda}}}\right]^{\frac{4 s \alpha}{(1+4 s)(1+2 \alpha)}}\left[m_{\alpha}\left(L_{\lambda}\right)+C\right]^{\frac{1}{1+2 \alpha}}\left[C_{1}+C_{2} \frac{1}{\varepsilon^{s+1 / 2}}(1+t)^{(s+1 / 2)(1 / \lambda-\beta)}\right]^{\frac{2 \alpha(1+2 s)}{(1+4 s)(1+2 \alpha)}} \\
& \leq C \varepsilon^{\gamma(s, \alpha)}(1+t)^{\delta(s, \alpha)}
\end{aligned}
$$

where $C>0$ depends on $\lambda, s$ and $\beta, \gamma(s, \alpha)=\frac{4 s^{2} \alpha-2 \alpha(1+2 s)^{2}}{2(1+4 s)(1+2 \alpha)}$ and $\delta(s, \alpha)=\frac{-4 s^{2} \alpha+2 \alpha(1+2 s)^{2}(1-\lambda \beta)}{2 \lambda(1+4 s)(1+2 \alpha)}$. By choosing $1-\lambda \beta$ small enough, we get $\delta(s, \alpha)<0$. Hence the result follows.

\subsection{Proofs of the Lemmas}

Proof of Lemma 10, We have

$$
\begin{aligned}
& d_{s}\left(\tilde{P}_{\lambda}(t), \tilde{P}_{\lambda, \varepsilon, r e g}(t)\right) \\
& =\sup _{\xi \neq 0} \frac{1}{|\xi|^{s}}\left|\mathrm{e}^{-|\xi|^{\lambda} \frac{t}{1+t}}-\left[\mathrm{e}^{-\varepsilon^{-\lambda} t\left(1-\widehat{M}_{\lambda, \varepsilon}\left(\frac{\xi}{(1+t)^{1 / \lambda}}\right)\right)}-\mathrm{e}^{-\varepsilon^{-\lambda} t}\left(1-\widehat{M}_{\lambda, \varepsilon}\left(\frac{\xi}{(1+t)^{1 / \lambda}}\right)\right)\right]\right| \\
& \leq \sup _{\xi \neq 0} \frac{1}{|\xi|^{s}}\left|\mathrm{e}^{-|\xi|^{\lambda} \frac{t}{1+t}}-\mathrm{e}^{-\varepsilon^{-\lambda} t\left(1-\widehat{M}_{\lambda, \varepsilon}\left(\frac{\xi}{(1+t)^{1 / \lambda}}\right)\right)}\right|+\sup _{\xi \neq 0} \frac{1}{|\xi|^{s}}\left|\mathrm{e}^{-\varepsilon^{-\lambda} t}\left(1-\widehat{M}_{\lambda, \varepsilon}\left(\frac{\xi}{(1+t)^{1 / \lambda}}\right)\right)\right| \\
& =I+I I .
\end{aligned}
$$


The term $I I$ can be written in the form

$$
I I=\sup _{\xi \neq 0} \frac{\left|\mathrm{e}^{-\varepsilon^{-\lambda} t}\left(1-\widehat{M}_{\lambda, \varepsilon}\left(\frac{\xi}{(1+t)^{1 / \lambda}}\right)\right)\right|}{|\xi|^{s}}=\frac{\mathrm{e}^{-\varepsilon^{-\lambda} t}}{(1+t)^{s / \lambda}} \sup _{\xi \neq 0} \frac{\left|1-\widehat{M}_{\lambda, \varepsilon}(\xi)\right|}{|\xi|^{s}} .
$$

Therefore, since $1<\lambda<2$, and $0<s<\lambda$ for $\xi \neq 0$ we get

$$
\frac{\left|1-\widehat{M}_{\lambda, \varepsilon}(\xi)\right|}{|\xi|^{s}}=\frac{\varepsilon^{\lambda}|\xi|^{\lambda}}{|\xi|^{s}\left(1+\varepsilon^{\lambda}|\xi|^{\lambda}\right)} \leq \varepsilon^{\lambda}|\xi|^{\lambda-s} .
$$

Thus, for any given positive constant $R>0$

$$
\sup _{\xi \neq 0} \frac{\left|1-\widehat{M}_{\lambda, \varepsilon}(\xi)\right|}{|\xi|^{s}} \leq \frac{1}{R^{s}}+\sup _{0<|\xi| \leq R} \frac{\left|1-\widehat{M}_{\lambda, \varepsilon}(\xi)\right|}{|\xi|^{s}} \leq \frac{1}{R^{s}}+\varepsilon^{\lambda} R^{\lambda-s} .
$$

By choosing $R=1 / \varepsilon$ we get

$$
I I \leq 2 \varepsilon^{s}
$$

Finally, we get the bound

$$
I I \leq C \varepsilon^{s} \frac{\mathrm{e}^{-\varepsilon^{-\lambda} t}}{(1+t)^{s / \lambda}} .
$$

The first term coincides with the term estimated in Proposition 5 . For this term we proved the bound

$$
I \leq C \varepsilon^{s / 2} \frac{t^{s /(2 \lambda)}}{(1+t)^{s / \lambda}} .
$$

This is enough to conclude.

Let us now estimate the moments.

Proof of Lemma 11. Let us remind that

$$
\tilde{P}_{\lambda}(v, t)=(1+t)^{1 / \lambda} P_{\lambda}\left((1+t)^{1 / \lambda} v, t\right)
$$

and that in (21) we stated that

$$
\int_{\mathbb{R}}|v|^{\alpha} P_{\lambda}(v, t) \mathrm{d} v=t^{\alpha / \lambda} m_{\alpha}\left(L_{\lambda}\right)
$$

Hence we obtain

$$
\int_{\mathbb{R}}|v|^{\alpha} \tilde{P}_{\lambda}(v, t) \mathrm{d} v=\int_{\mathbb{R}}|v|^{\alpha}(1+t)^{1 / \lambda} P_{\lambda}\left((1+t)^{1 / \lambda} v, t\right) \mathrm{d} v=\frac{1}{(1+t)^{\alpha / \lambda}} \int_{\mathbb{R}}|v|^{\alpha} P_{\lambda}(v, t) \mathrm{d} v \leq m_{\alpha}\left(L_{\lambda}\right) .
$$

To estimate $m_{\alpha}\left(\tilde{P}_{\lambda, \varepsilon, r e g}(t)\right)$, we remark that, thanks to expression (42), it is enough to prove that

$$
\int_{\mathbb{R}}|v|^{\alpha}\left(\mathrm{e}^{-\varepsilon^{-\lambda} t} \sum_{n=1}^{+\infty}\left(\frac{t}{\varepsilon^{\lambda}}\right)^{n} \frac{1}{n !} M_{\lambda, \varepsilon}^{* n}(v)\right) \mathrm{d} v \leq t^{\alpha / \lambda} C .
$$

By definition

$$
M_{\lambda, \varepsilon}^{* n}(v)=\varepsilon^{-1} M_{\lambda}^{* n}\left(\varepsilon^{-1} v\right)
$$

and this implies

$$
\int_{\mathbb{R}}|v|^{\alpha} M_{\lambda, \varepsilon}^{* n}(v) \mathrm{d} v=\varepsilon^{\alpha} \int_{\mathbb{R}}|v|^{\alpha} M_{\lambda}^{* n}(v) \mathrm{d} v .
$$


Now, consider that $n^{1 / \lambda} M_{\lambda}^{* n}\left(n^{1 / \lambda} v\right)$ is the density of the sum $\left(X_{1}+\cdots+X_{n}\right) / n^{1 / \lambda}$, where $\left(X_{n}\right)_{n \geq 1}$ is a sequence of independent and identically distributed real-valued random variables with common density $M_{\lambda}$ in the domain of normal attraction of $L_{\lambda}(v) \mathrm{d} v$. This fact can be used to write

$$
\int_{\mathbb{R}}|v|^{\alpha} M_{\lambda}^{* n}(v) \mathrm{d} v=n^{\alpha / \lambda} \int_{\mathbb{R}}|w|^{\alpha} n^{1 / \lambda} M_{\lambda}^{* n}\left(n^{1 / \lambda} w\right) \mathrm{d} w .
$$

Since $M_{\lambda}$ is a centered distribution, $\left(X_{1}+\cdots+X_{n}\right) / n^{1 / \lambda}$ converges in law to $L_{\lambda}(v) \mathrm{d} v$. Therefore, thanks to Lemma 5.2.2 in IL71] (see Proposition 3) one obtains

$$
\int_{\mathbb{R}}|w|^{\alpha} n^{1 / \lambda} M_{\lambda}^{* n}\left(n^{1 / \lambda} w\right) \mathrm{d} w \leq C,
$$

and this implies

$$
\int_{\mathbb{R}}|v|^{\alpha} M_{\lambda, \varepsilon}^{* n}(v) \mathrm{d} v \leq C \varepsilon^{\alpha} n^{\alpha / \lambda}
$$

In conclusion

$$
\begin{aligned}
\int_{\mathbb{R}}|v|^{\alpha}\left(\mathrm{e}^{-\varepsilon^{-\lambda} t} \sum_{n=1}^{+\infty}\left(\frac{t}{\varepsilon^{\lambda}}\right)^{n} \frac{1}{n !} M_{\lambda, \varepsilon}^{* n}(v)\right) \mathrm{d} v & =\mathrm{e}^{-\varepsilon^{-\lambda} t} \sum_{n=1}^{+\infty}\left(\frac{t}{\varepsilon^{\lambda}}\right)^{n} \frac{1}{n !} \int_{\mathbb{R}}|v|^{\alpha} M_{\lambda, \varepsilon}^{* n}(v) \mathrm{d} v \\
& \leq C \varepsilon^{\alpha} \mathrm{e}^{-\varepsilon^{-\lambda}} t \sum_{n=1}^{+\infty}\left(\frac{t}{\varepsilon^{\lambda}}\right)^{n} \frac{1}{n !} n^{\alpha / \lambda} .
\end{aligned}
$$

We will now prove that

$$
\mathrm{e}^{-\varepsilon^{-\lambda} t} \sum_{n=1}^{+\infty}\left(\frac{t}{\varepsilon^{\lambda}}\right)^{n} \frac{1}{n !} n^{\alpha / \lambda} \leq \frac{t^{\alpha / \lambda}}{\varepsilon^{\alpha}}
$$

or, in an equivalent way

$$
\sum_{n=1}^{+\infty}\left(\frac{t}{\varepsilon^{\lambda}}\right)^{n} \frac{1}{n !}\left(\frac{n}{t}\right)^{\alpha / \lambda} \leq \frac{\mathrm{e}^{\varepsilon^{-\lambda} t}}{\varepsilon^{\alpha}}
$$

If $\tau=\varepsilon^{-\lambda} t$, and $\beta=\alpha / \lambda$, this amounts to prove that, if $0<\beta<1$

$$
\sum_{n=1}^{+\infty} \frac{\tau^{n}}{n !}\left(\frac{n}{\tau}\right)^{\beta} \leq \mathrm{e}^{\tau}
$$

To this end, it is enough to remark that, if $\beta=0$

$$
\sum_{n=1}^{+\infty} \frac{\tau^{n}}{n !}=\mathrm{e}^{\tau}-1
$$

On the other side, if $\beta=1$ we get

$$
\sum_{n=1}^{+\infty} \frac{\tau^{n}}{n !}\left(\frac{n}{\tau}\right)=\sum_{n=0}^{+\infty} \frac{\tau^{n}}{n !}=\mathrm{e}^{\tau}
$$

Hence, if $0<\beta<1$

$$
\sum_{n=1}^{+\infty} \frac{\tau^{n}}{n !}\left(\frac{n}{\tau}\right)^{\beta} \leq \sum_{n=1}^{+\infty} \frac{\tau^{n}}{n !} \max \left(1, \frac{n}{\tau}\right) \leq \max \left(\sum_{n=1}^{+\infty} \frac{\tau^{n}}{n !}, \sum_{n=0}^{+\infty} \frac{\tau^{n}}{n !}\right)=\mathrm{e}^{\tau} .
$$

All these bounds imply

$$
\int_{\mathbb{R}}|v|^{\alpha} \tilde{P}_{\lambda, \varepsilon, r e g}(v, t) \mathrm{d} v \leq \frac{t^{\alpha / \lambda}}{(1+t)^{\alpha / \lambda}} C \leq C .
$$




\section{Remark 13}

$A$ direct consequence of this Lemma is that the moment of order $\alpha$ of the approximated solution $g_{\varepsilon}(t)$ of equation (26), grows at a polynomial rate in time in correspondence to any generic centered distribution $M_{\lambda}(v) \mathrm{d} v$ belonging to the domain of normal attraction of the stable law $L_{\lambda}(v) \mathrm{d} v$

$$
\int_{\mathbb{R}}|v|^{\alpha} g_{\varepsilon}(v, t) \mathrm{d} v \leq C t^{\alpha / \lambda} .
$$

Proof of Lemma 12, By definition

$$
\tilde{P}_{\lambda}(v, t)=\left(\frac{1+t}{t}\right)^{1 / \lambda} L_{\lambda}\left(\left(\frac{1+t}{t}\right)^{1 / \lambda} v\right) .
$$

Therefore, for $t>0$ large enough and $s>0$

$$
\left\|\tilde{P}_{\lambda}(t)\right\|_{\dot{H}^{s}}=\left(\frac{1+t}{t}\right)^{(s+1 / 2) / \lambda}\left\|L_{\lambda}\right\|_{\dot{H}^{s}} \leq C_{1} .
$$

Expressions (41) and (28) lead to

$$
\mathcal{F}\left(\tilde{P}_{\lambda, \varepsilon, r e g}\right)(\xi, t)=\mathrm{e}^{-\varepsilon^{-\lambda} t}\left[\exp \left(\varepsilon^{-\lambda} t \widehat{M}_{\lambda, \varepsilon}\left(\frac{\xi}{(1+t)^{1 / \lambda}}\right)\right)-1+\widehat{M}_{\varepsilon, \lambda}\left(\frac{\xi}{(1+t)^{1 / \lambda}}\right)\right] .
$$

Therefore

$$
\begin{aligned}
& \left\|\tilde{P}_{\lambda, \varepsilon, \text { reg }}(t)\right\|_{\dot{H}^{s}}^{2} \leq C \int_{\mathbb{R}}|\xi|^{2 s} \exp \left(-2 t \varepsilon^{-\lambda}\right)\left(\exp \left(\varepsilon^{-\lambda} t \widehat{M}_{\lambda, \varepsilon}\left(\frac{\xi}{(1+t)^{1 / \lambda}}\right)\right)-1\right)^{2} \mathrm{~d} \xi+ \\
& \int_{\mathbb{R}}|\xi|^{2 s} \exp \left(-2 t \varepsilon^{-\lambda}\right) \widehat{M}_{\lambda, \varepsilon}\left(\frac{\xi}{(1+t)^{1 / \lambda}}\right)^{2} \mathrm{~d} \xi=A+B .
\end{aligned}
$$

By a change of variable, term $B$ can be estimated as follows

$$
\begin{aligned}
B & =(1+t)^{(2 s+1) / \lambda} \exp \left(-2 t \varepsilon^{-\lambda}\right)\left\|M_{\lambda, \varepsilon}\right\|_{\dot{H}^{s}}^{2}=(1+t)^{(2 s+1) / \lambda} \frac{\exp \left(-2 t \varepsilon^{-\lambda}\right)}{\varepsilon^{2 s+1}}\left\|M_{\lambda}\right\|_{\dot{H}^{s}}^{2} \\
& \leq C_{\lambda, s}(1+t)^{(2 s+1) / \lambda} \frac{\exp \left(-2 t \varepsilon^{-\lambda}\right)}{\varepsilon^{2 s+1}}
\end{aligned}
$$

for $s<\lambda-1 / 2$.

Likewise, the term $A$ can be expressed as

$$
A=(1+t)^{(2 s+1) / \lambda} \int_{\mathbb{R}}|\eta|^{2 s}\left(\exp \left(-t \varepsilon^{-\lambda}\left(1-\widehat{M}_{\lambda, \varepsilon}(\eta)\right)\right)-\exp \left(-t \varepsilon^{-\lambda}\right)\right)^{2} \mathrm{~d} \eta=(1+t)^{(2 s+1) / \lambda} I_{\varepsilon}(t) .
$$

To estimate the term $I_{\varepsilon}(t)$, consider that it satisfies the differential equation

$$
\frac{\mathrm{d} I_{\varepsilon}(t)}{\mathrm{d} t}=-2 \varepsilon^{-\lambda} I_{\varepsilon}(t)+2 \varepsilon^{-\lambda} A_{\varepsilon}(t),
$$

where $A_{\varepsilon}(t)$ can bounded in a precise way. In fact

$$
\begin{aligned}
& \frac{\mathrm{d} I_{\varepsilon}(t)}{\mathrm{d} t}=\int_{\mathbb{R}}|\eta|^{2 s} 2\left(\exp \left(-t \varepsilon^{-\lambda}\left(1-\widehat{M}_{\lambda, \varepsilon}(\eta)\right)\right)-\exp \left(-t \varepsilon^{-\lambda}\right)\right) \times \\
& \left(-\exp \left(-t \varepsilon^{-\lambda}\left(1-\widehat{M}_{\lambda, \varepsilon}(\eta)\right)\right) \frac{1-\widehat{M}_{\lambda, \varepsilon}(\eta)}{\varepsilon^{\lambda}}+\frac{1}{\varepsilon^{\lambda}} \exp \left(-t \varepsilon^{-\lambda}\right)\right) \mathrm{d} \eta \\
= & -2 \varepsilon^{-\lambda} \int_{\mathbb{R}}|\eta|^{2 s}\left(\exp \left(-t \varepsilon^{-\lambda}\left(1-\widehat{M}_{\lambda, \varepsilon}(\eta)\right)\right)-\exp \left(-t \varepsilon^{-\lambda}\right)\right)^{2} \mathrm{~d} \eta+ \\
& 2 \varepsilon^{-\lambda} \int_{\mathbb{R}}|\eta|^{2 s}\left(\exp \left(-t \varepsilon^{-\lambda}\left(1-\widehat{M}_{\lambda, \varepsilon}(\eta)\right)\right)-\exp \left(-t \varepsilon^{-\lambda}\right)\right) \widehat{M}_{\lambda, \varepsilon}(\eta) \exp \left(-t \varepsilon^{-\lambda}\left(1-\widehat{M}_{\lambda, \varepsilon}(\eta)\right)\right) \mathrm{d} \eta \\
& =-2 \varepsilon^{-\lambda} I_{\varepsilon}(t)+2 \varepsilon^{-\lambda} A_{\varepsilon}(t) .
\end{aligned}
$$


It remains to estimate the term $A_{\varepsilon}(t)$. For a given $\beta>0$, we split the $A_{\varepsilon}(t)$ term into two terms

$$
\begin{aligned}
A_{\varepsilon}(t)= & \int_{\mathbb{R}}|\eta|^{2 s} \widehat{M}_{\lambda, \varepsilon}(\eta) \exp \left(-2 t \varepsilon^{-\lambda}\left(1-\widehat{M}_{\lambda, \varepsilon}(\eta)\right)\right)\left(1-\exp \left(-t \varepsilon^{-\lambda} \widehat{M}_{\lambda, \varepsilon}(\eta)\right)\right) \mathrm{d} \eta \\
= & \int_{0 \leq|\eta| \leq \frac{1}{(1+t)^{\beta}}}|\eta|^{2 s} \widehat{M}_{\lambda, \varepsilon}(\eta) \exp \left(-2 t \varepsilon^{-\lambda}\left(1-\widehat{M}_{\lambda, \varepsilon}(\eta)\right)\right)\left(1-\exp \left(-t \varepsilon^{-\lambda} \widehat{M}_{\lambda, \varepsilon}(\eta)\right)\right) \mathrm{d} \eta+ \\
& \int_{|\eta|>\frac{1}{(1+t)^{\beta}}}|\eta|^{2 s} \widehat{M}_{\lambda, \varepsilon}(\eta) \exp \left(-2 t \varepsilon^{-\lambda}\left(1-\widehat{M}_{\lambda, \varepsilon}(\eta)\right)\right)\left(1-\exp \left(-t \varepsilon^{-\lambda} \widehat{M}_{\lambda, \varepsilon}(\eta)\right)\right) \mathrm{d} \eta \\
= & A_{1}+A_{2} .
\end{aligned}
$$

Since $0<\widehat{M}_{\lambda, \varepsilon}(\eta) \leq 1$, we have

$$
A_{1} \leq \int_{0 \leq|\eta| \leq \frac{1}{(1+t)^{\beta}}}|\eta|^{2 s} \mathrm{~d} \eta=\frac{2}{2 s+1} \frac{1}{(1+t)^{\beta(2 s+1)}}=\frac{C_{s}}{(1+t)^{\beta(2 s+1)}} .
$$

Moreover

$$
\begin{aligned}
A_{2} & =\int_{|\eta|>\frac{1}{(1+t)^{\beta}}}|\eta|^{2 s} \widehat{M}_{\lambda, \varepsilon}(\eta) \exp \left(-2 t \varepsilon^{-\lambda}\left(1-\widehat{M}_{\lambda, \varepsilon}(\eta)\right)\right)\left(1-\exp \left(-t \varepsilon^{-\lambda} \widehat{M}_{\lambda, \varepsilon}(\eta)\right)\right) \mathrm{d} \eta \\
& \leq \sup _{|\eta|>\frac{1}{(1+t)^{\beta}}} \exp \left(-2 t \varepsilon^{-\lambda}\left(1-\widehat{M}_{\lambda, \varepsilon}(\eta)\right)\right) \int_{|\eta|>\frac{1}{(1+t)^{\beta}}}|\eta|^{2 s} \widehat{M}_{\lambda, \varepsilon}(\eta) \mathrm{d} \eta \\
& =C_{\lambda, s} \frac{1}{\varepsilon^{2 s+1}} \sup _{|\eta|>\frac{1}{(1+t)^{\beta}}} \exp \left(-2 t \varepsilon^{-\lambda}\left(1-\widehat{M}_{\lambda, \varepsilon}(\eta)\right)\right)
\end{aligned}
$$

where $C_{\lambda, s}=\int_{\mathbb{R}}|\eta|^{2 s} \widehat{M}_{\lambda}(\eta) \mathrm{d} \eta<+\infty$ for $s<(\lambda-1) / 2$. Now, since $\widehat{M}_{\lambda, \varepsilon}(\eta)=\frac{1}{1+|\varepsilon \xi|^{\lambda}}$ we obtain

$$
\sup _{|\eta|>\frac{1}{(1+t)^{\beta}}} \exp \left(-2 t \varepsilon^{-\lambda}\left(1-\widehat{M}_{\lambda, \varepsilon}(\eta)\right)\right)=\sup _{|\eta|>\frac{1}{(1+t)^{\beta}}} \exp \left(-\frac{2 t|\eta|^{\lambda}}{1+\varepsilon^{\lambda}|\eta|^{\lambda}}\right) .
$$

Since the function $\exp \left(-\frac{2 t|\eta|^{\lambda}}{1+\varepsilon^{\lambda}|\eta|^{\lambda}}\right)$ is decreasing in $\eta$, we get

$$
\sup _{|\eta|>\frac{1}{(1+t)^{\beta}}} \exp \left(-\frac{2 t|\eta|^{\lambda}}{1+\varepsilon^{\lambda}|\eta|^{\lambda}}\right)=\exp \left(-2 t \frac{\frac{1}{(1+t)^{\beta \lambda}}}{1+\varepsilon^{\lambda} \frac{1}{(1+t)^{\beta \lambda}}}\right)=\exp \left(-\frac{2 t}{\varepsilon^{\lambda}+(1+t)^{\beta \lambda}}\right) .
$$

If $0<\beta<1 / \lambda$, we conclude that there exists a constant $C_{\beta}>0$ such that

$$
\exp \left(-\frac{2 t}{\varepsilon^{\lambda}+(1+t)^{\beta \lambda}}\right) \leq \mathrm{e}^{-C_{\beta} t^{1-\beta \lambda}} .
$$

In the end, for $t>0$ and $0<\beta<1 / \lambda$ there exists a constant $C=C(\lambda, s, \beta)>0$ such that

$$
A_{\varepsilon}(t) \leq\left(\frac{C_{s}}{(1+t)^{\beta(2 s+1)}}+\frac{C_{\lambda, s}}{\varepsilon^{2 s+1}} \mathrm{e}^{-C_{\beta} t^{1-\beta \lambda}}\right) \leq \frac{C}{\varepsilon^{2 s+1}} \frac{1}{(1+t)^{\beta(2 s+1)}} .
$$

With this estimate we can study the differential relation

$$
\frac{\mathrm{d} I_{\varepsilon}(t)}{\mathrm{d} t}=-2 \varepsilon^{-\lambda} I_{\varepsilon}(t)+2 \varepsilon^{-\lambda} A_{\varepsilon}(t) .
$$

For $a>0$ to be suitably chosen, we get

$$
I_{\varepsilon}(t) \mathrm{e}^{2 \varepsilon^{-\lambda} t}-I_{\varepsilon}\left(a \varepsilon^{\lambda}\right) \mathrm{e}^{2 a}=2 \varepsilon^{-\lambda} \int_{a \varepsilon^{\lambda}}^{t} A_{\varepsilon}(\sigma) \mathrm{e}^{2 \varepsilon^{-\lambda} \sigma} \mathrm{d} \sigma .
$$


Since, for $0<s<\lambda-1 / 2$,

$$
\begin{aligned}
I_{\varepsilon}\left(a \varepsilon^{\lambda}\right) & =\int_{\mathbb{R}}|\eta|^{2 s}\left(\mathrm{e}^{-a\left(1-\widehat{M}_{\lambda, \varepsilon}(\eta)\right)}-\mathrm{e}^{-a}\right)^{2} \mathrm{~d} \eta=\frac{\mathrm{e}^{-2 a}}{\varepsilon^{2 s+1}} \int_{\mathbb{R}}|\xi|^{2 s}\left(\mathrm{e}^{\frac{a}{1+|\xi| \lambda}}-1\right)^{2} \mathrm{~d} \xi \\
& =C_{s, a} \frac{1}{\varepsilon^{2 s+1}}<+\infty,
\end{aligned}
$$

for $t \geq a \geq a \varepsilon^{\lambda}$ we get

$$
I_{\varepsilon}(t)=I_{\varepsilon}\left(a \varepsilon^{\lambda}\right) \mathrm{e}^{-2 \varepsilon^{-\lambda}\left(t-a \varepsilon^{\lambda}\right)}+2 \varepsilon^{-\lambda} \int_{a \varepsilon^{\lambda}}^{t} A_{\varepsilon}(\sigma) \mathrm{e}^{-2 \varepsilon^{-\lambda}(t-\sigma)} \mathrm{d} \sigma .
$$

Thanks to estimate (47) we get

$$
\int_{a \varepsilon^{\lambda}}^{t} A_{\varepsilon}(\sigma) \mathrm{e}^{-2 \varepsilon^{-\lambda}(t-\sigma)} \mathrm{d} \sigma \leq \frac{C}{\varepsilon^{2 s+1}} \int_{a \varepsilon^{\lambda}}^{t} \frac{\mathrm{e}^{-2 \varepsilon^{-\lambda}(t-\sigma)}}{\sigma^{\beta(2 s+1)}} \mathrm{d} \sigma .
$$

Integrating by parts

$$
\begin{aligned}
\int_{a \varepsilon^{\lambda}}^{t} \frac{\mathrm{e}^{-2 \varepsilon^{-\lambda}(t-\sigma)}}{\sigma^{\beta(2 s+1)}} \mathrm{d} \sigma & =\frac{\varepsilon^{\lambda}}{2} \frac{1}{t^{\beta(2 s+1)}}-\frac{\varepsilon^{\lambda}}{2} \frac{\mathrm{e}^{-2 \varepsilon^{-\lambda}\left(t-a \varepsilon^{\lambda}\right)}}{a^{\beta(2 s+1)} \varepsilon^{\lambda \beta(2 s+1)}}+\frac{\varepsilon^{\lambda}}{2} \beta(2 s+1) \int_{a \varepsilon^{\lambda}}^{t} \frac{\mathrm{e}^{-2 \varepsilon^{-\lambda}(t-\sigma)}}{\sigma^{\beta(2 s+1)+1}} \mathrm{~d} \sigma \\
& \leq \frac{\varepsilon^{\lambda}}{2} \frac{1}{t^{\beta(2 s+1)}}+\frac{\varepsilon^{\lambda}}{2} \frac{\beta(2 s+1)}{a \varepsilon^{\lambda}} \int_{a \varepsilon^{\lambda}}^{t} \frac{\mathrm{e}^{-2 \varepsilon^{-\lambda}(t-\sigma)}}{\sigma^{\beta(2 s+1)}} \mathrm{d} \sigma .
\end{aligned}
$$

Let us choose $a$ such that $1-\frac{\beta(2 s+1)}{2 a}>0(a$ depends on $s$ and $\beta)$. We obtain

$$
\int_{a \varepsilon^{\lambda}}^{t} \frac{\mathrm{e}^{-2 \varepsilon^{-\lambda}(t-\sigma)}}{\sigma^{\beta(2 s+1)}} \mathrm{d} \sigma \leq C_{s, \beta} \frac{\varepsilon^{\lambda}}{t^{\beta(2 s+1)}} .
$$

Getting back to (48), (49) and (50), we proved that there exists a constant $C=C(\lambda, s, \beta)>0$ such that

$$
I_{\varepsilon}(t) \leq C \frac{1}{\varepsilon^{2 s+1}}\left(\mathrm{e}^{-2 t \varepsilon^{-\lambda}}+\frac{1}{t^{\beta(2 s+1)}}\right) .
$$

Finally, by (45), (46) and (51), we get

$$
\left\|\tilde{P}_{\lambda, \varepsilon, \text { reg }}(t)\right\|_{\dot{H}^{s}}^{2} \leq C \frac{(1+t)^{(2 s+1) / \lambda}}{\varepsilon^{2 s+1}}\left(\mathrm{e}^{-2 t \varepsilon^{-\lambda}}+\frac{1}{t^{\beta(2 s+1)}}\right) \leq C \frac{1}{\varepsilon^{2 s+1}}(1+t)^{(2 s+1)(1 / \lambda-\beta)}
$$

and denoting $C_{2}=C$

$$
\left\|\tilde{P}_{\lambda, \varepsilon, r e g}(t)\right\|_{\dot{H}^{s}} \leq C_{2} \frac{1}{\varepsilon^{s+1 / 2}}(1+t)^{(s+1 / 2)(1 / \lambda-\beta)} .
$$

\section{Conclusions}

In this paper we studied an approximation to fractional diffusion equations obtained by using the argument originally proposed for the linear diffusion equation by Rosenau Ros92. As it happens for the linear diffusion, the approximation coincides with a linear kinetic equation of Boltzmann type, in which the Maxwellian background is now represented by a Linnik distribution [Lin53, Lin62. A detailed analysis of the solution to this kinetic equation allows us to obtain various interesting properties. Among others, it was interesting to discover that the solution to the Rosenau approximation can be split into two parts, easily identified in terms of their regularity: one singular, and the other regular. The former simply represents a perturbation of mass zero, and it decays to zero exponentially both with respect to time and to the small parameter $\varepsilon$ characterizing the approximation. The latter is shown to approach in time, for any fixed value of the parameter $\varepsilon$, the fundamental solution to the 
fractional diffusion equation in strong sense. This allows us to conclude that the Rosenau argument introduces in a natural way a consistent approximation of fractional diffusion equations, which not only reproduces the limit phenomenon at fixed time and for small values of the parameter $\varepsilon$, but also reproduces, apart of rapidly decaying perturbations, the limit phenomenon for large times. Out of doubts, these results could be fruitfully employed to construct new numerical approximations to fractional diffusion equations.

Acknowledgements. This work has been written within the activities of the National Group of Mathematical Physics (GNFM) and of the National Group of Mathematical Analysis, Probability and Applications (GNAMPA) of INDAM. Two authors (AP) and (GT) acknowledge support by MIUR project "Optimal mass transportation, geometrical and functional inequalities with applications". We thank Federico Bassetti for useful discussions about the domain of normal attraction of a stable law.

\section{References}

[AA93] D. N. Anderson and B. C. Arnold, Linnik distributions and processes, J. Appl. Probab. 30 (1993), no. 2, 330-340.

[BBDE11] J-P. Bartier, A. Blanchet, J. Dolbeault and M. Escobedo, Improved intermediate asymptotics for the heat equation, Appl. Math. Letters 24 (2011), 76-81.

[BLM11] F. Bassetti, L. Ladelli and D. Matthes, Central limit theorem for a class of onedimensional kinetic equations, Probab. Theory Related Fields, 150 (2011), 77-109.

[BLR08] F. Bassetti, L. Ladelli and E. Regazzini, Probabilistic study of the speed of approach to equilibrium for an inelastic Kac model, J. Stat. Phys., 133, (2008), 4, 683-710.

[BWM00] D.A. Benson, S. W. Wheatcraft, and M. M. Meerschaert, The fractional-order governing equation of Lévy motion, Water Resources Research 36 (2000), no. 6, 1413-1423.

[Cer88] C. Cercignani, The Boltzmann equation and its applications, Applied Mathematical Sciences, vol. 67, Springer-Verlag, New York, 1988.

[CGT99] E. A. Carlen, E. Gabetta, and G. Toscani, Propagation of smoothness and the rate of exponential convergence to equilibrium for a spatially homogeneous Maxwellian gas, Comm. Math. Phys. 199 (1999), no. 3, 521-546.

[CT07] J.A. Carrillo, G. Toscani, Contractive probability metrics ans asymptotic behavior of dissipative kinetic equations (Notes of the Porto Ercole School, June 2006), Riv. Mat. Univ. Parma, 7 6, (2007) 75-198.

[Cha98] A. S. Chaves, A fractional diffusion equation to describe Lévy flights, Phys. Lett. A 239 (1998), no. 1-2, 13-16.

[Fel71] W. Feller, An introduction to probability theory and its applications. Vol. II., Second edition, John Wiley \& Sons Inc., New York, 1971.

[FPTT12] G. Furioli, A. Pulvirenti, E. Terraneo, and G. Toscani, The grazing collision limit of the inelastic Kac model around a Lévy-type equilibrium, SIAM J. Math. Anal. 44 (2012), no. 2, $827-850$.

[GM98] R. Gorenko, F. Mainardi, Fractional calculus and stable probability distributions, Arch. Mech. 50 (1998), 377-388. 
[GTW95] E. Gabetta, G. Toscani, B. Wennberg, Metrics for probability distributions and the trend to equilibrium for solutions of the Boltzmann equation, J. Statist. Phys. 81 (1995), 901-934.

[IL71] I. A. Ibragimov, and Yu. V. Linnik, Independent and stationary sequences of random variables, With a supplementary chapter by I. A. Ibragimov and v. V. Petrov, Translation from the Russian edited by J. F. C. Kingman, Wolters-Noordhoff Publishing, Groningen, 1971, 443 pages.

[KZS97] J. Klafter, G. Zumofen, and M.F. Shlesinger, Long-tailed distributions and non-Brownian transport in complex systems in: F. Mallamace, H.E. Stanley (Eds.), The Physics of Complex Systems, IOS Press, Amsterdam, 1997, 85-94

[KOH95] S. Kotz, I. V. Ostrovskiü, and A. Hayfavi, Analytic and asymptotic properties of Linnik's probability densities. I, J. Math. Anal. Appl. 193 (1995), no. 1, 353-371.

[LB10] Z. Lin and Z. Bai, Probability inequalities, Science Press Beijing, Beijing, 2010.

[Lin53] Yu. V. Linnik, Linear forms and statistical criteria. II, Ukrain. Mat. Žurnal 5 (1953), 247-290.

[Lin62] Ju. V. Linnik, Linear forms and statistical criteria. I,II, Selected Transl. Math. Statist. and Prob., Vol. 3, Amer. Math. Soc., Providence, R.I., 1962, pp. 1-90.

[LR79] R. G. Laha and v. K. Rohatgi, Probability theory, John Wiley \& Sons, New YorkChichester-Brisbane, 1979, Wiley Series in Probability and Mathematical Statistics.

[LT01] H. Liu and E. Tadmor, Critical thresholds in a convolution model for nonlinear conservation laws, SIAM J. Math. Anal. 33 (2001), no. 4, 930-945.

[MFL02] F. J. Molz, III, G. J. Fix, III, and S. Lu, A physical interpretation for the fractional derivative in Levy diffusion, Appl. Math. Lett. 15 (2002), no. 7, 907-911.

[MMM11] A. Mellet, S. Mischler, and C. Mouhot, Fractional diffusion limit for collisional kinetic equations, Arch. Ration. Mech. Anal. 199 (2011), no. 2, 493-525.

[PT13] L. Pareschi, and G. Toscani, Interacting multiagent systems. Kinetic equations \& Monte carlo methods Oxford University Press, Oxford (2013)

[Pól23] G. Pólya, On the zeros of an integral function represented by Fourier's integral, Mess. Math. 52 (1923), 185-188.

[PT04] A. Pulvirenti, and G. Toscani, Asymptotic properties of the inelastic Kac model. J. Statist. Phys., 114(2004), 1453?1480.

[RT13] T. Rey and G. Toscani, Large-time behavior of the solutions to Rosenau type approximations to the heat equation, In press, SIAM J. Appl. Math., 2013.

[Ros92] P. Rosenau, Tempered diffusion: A transport process with propagating fronts and inertial delay, Physical Review A 46 (1992), no. 12, 12-15.

[Ros95] P. Rosenau, Fast and superfast diffusion processes. Phys. Rev. Lett, vol. 74, (1995) 10561059 .

[SBMW01] R. Schumer, D. A. Benson, M.M. Meerschaert, and S.W. Wheatcraft, Eulerian derivation of the fractional advection-dispersion equation, Journal of Contaminant Hydrology 48 (2001), 69-88.

[ST92] S. Schochet and E. Tadmor, The regularized Chapman-Enskog expansion for scalar conservation laws, Arch. Rational Mech. Anal. 119 (1992), no. 2, 95-107.

[Tos96] G. Toscani, Kinetic approach to the asymptotic behaviour of the solution to diffusion equations, Rend. Mat. Appl. (7) 16 (1996), no. 2, 329-346. 
[Wil51] E. Wild, On Boltzmann's equation in the kinetic theory of gases, Proc. Cambridge Philos. Soc. 47 (1951), 602-609.

[Vaz07] J.L. Vázquez, The Porous Medium Equation: Mathematical Theory, Oxford, UK, Oxford University Press, 2007

[Vaz11] J.L. Vázquez, From Newton's equation to fractional diffusion and wave equations, Advances in Difference Equations, ID 169421, (2011),13 pages. 